\title{
NOD2 downregulates colonic inflammation by IRF4-mediated inhibition of K63-linked polyubiquitination of RICK and TRAF6
}

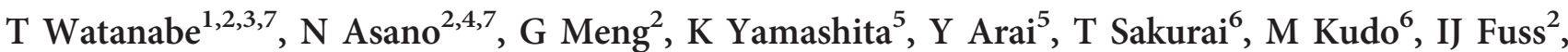 \\ A Kitani ${ }^{2}$, T Shimosegawa ${ }^{4}, \mathrm{~T} \mathrm{Chiba}^{3}$ and $\mathrm{W}$ Strober ${ }^{2}$
}

It is well established that polymorphisms of the caspase activation and recruitment domain 15 (CARD15) gene, a major risk factor in Crohn's disease (CD), lead to loss of nucleotide-binding oligomerization domain 2 (NOD2) function. However, a molecular explanation of how such loss of function leads to increased susceptibility to CD has remained unclear. In a previous study exploring this question, we reported that activation of NOD2 in human dendritic cells by its ligand, muramyl dipeptide (MDP), negatively regulates Toll-like receptor (TLR)-mediated inflammatory responses. Here we show that NOD2 activation results in increased interferon regulatory factor 4 (IRF4) expression and binding to tumor necrosis factor receptor associated factor 6 (TRAF6) and RICK (receptor interacting serine-threonine kinase). We then show that such binding leads to IRF4-mediated inhibition of Lys63-linked polyubiquitination of TRAF6 and RICK and thus to downregulation of nuclear factor (NF)-кB activation. Finally, we demonstrate that protection of mice from the development of experimental colitis by MDP or IRF4 administration is accompanied by similar IRF4-mediated effects on polyubiquitination of TRAF6 and RICK in colonic lamina propria mononuclear cells. These findings thus define a mechanism of NOD2-mediated regulation of innate immune responses to intestinal microflora that could explain the relation of CARD15 polymorphisms and resultant NOD2 dysfunction to CD.

\section{INTRODUCTION}

Nucleotide-binding oligomerization domain 2 (NOD2) is a NOD-like receptor (NLR) family member that functions as an intracellular sensor of small peptides (such as muramyl dipeptide (MDP)) derived from the peptidoglycan component of the bacterial cell wall. ${ }^{1,2}$ Activation of NOD2 occurring upon sensing of its ligand through its leucine-rich repeat domain is followed by NOD2 oligomerization and exposure of its caspase activation and recruitment domain (CARD). This enables a physical interaction between NOD2 and a downstream adaptor molecule, receptor interacting serine-threonine kinase (RICK) that then results in lysine 63 (K63)-linked polyubiquitination of RICK. ${ }^{1-3}$ Ubiquitinated RICK then interacts with transforming growth factor- $\beta$-activated kinase 1 and tumor necrosis factor receptor associated factor 6 (TRAF6) to cause nuclear translocation of nuclear factor (NF)- $\mathrm{\kappa B}$ subunits. ${ }^{4-8}$

The functional importance of NOD2 is underscored by the fact that polymorphisms in the CARD15 gene encoding NOD2 are associated with Crohn's disease (CD) and other immune disorders such as graft versus host disease. ${ }^{1,2,9}$ However, despite extensive investigation, the molecular mechanisms by which such polymorphisms contribute to these diseases are not completely understood. ${ }^{1,9}$ A possible clue to the nature of these mechanisms comes from the fact that MDP activation of NOD2 can both positively and negatively regulate Toll-like receptor (TLR)-mediated inflammatory responses. For example, synergistic production of proinflammatory cytokine responses has been observed in human antigen-presenting cells (APCs) upon

\footnotetext{
${ }^{1}$ Center for Innovation in Immunoregulative Technology and Therapeutics, Kyoto University Graduate School of Medicine, Kyoto, Japan. ${ }^{2}$ Mucosal Immunity Section, Laboratory of Host Defenses, National Institute of Allergy and Infectious Diseases, National Institutes of Health, Bethesda, Maryland, USA. ${ }^{3}$ Department of Gastroenterology and Hepatology, Kyoto University Graduate School of Medicine, Kyoto, Japan. ${ }^{4}$ Division of Gastroenterology, Tohoku University Graduate School of Medicine, Sendai, Japan. ${ }^{5}$ Department of Hematology and Oncology, Kyoto University Graduate School of Medicine, Kyoto, Japan and ${ }^{6}$ Department of Gastroenterology and Hepatology, Kinki University School of Medicine, Osakasayama, Japan. Correspondence: T Watanabe or W Strober (tmhrwtnb@kuhp.kyoto-u.ac.jp or wstrober@niaid.nih.gov)

${ }^{7}$ The first two authors contributed equally to this work.
} 
simultaneous stimulation of MDP and TLR ligands. ${ }^{10,11}$ This synergism could contribute to the control of the gastrointestinal commensal microflora that is necessary for the prevention of $\mathrm{CD} .^{12}$

However, the above described capacity of NOD2 activation to augment innate immune responses is accompanied by the fact that such activation also has a negative effect on TLR signaling. Thus, we and others have shown that preactivation of NOD2 by MDP induces decreased proinflammatory cytokine responses in human APCs upon subsequent challenge with TLR ligands. ${ }^{13-17}$ In addition, Hedl et al. ${ }^{15,16}$ have shown that such tolerogenic responses were not observed in human APCs from patients bearing CD-associated NOD2 mutations. ${ }^{17} \mathrm{We}$ have also found that systemic injection of MDP protects NOD2-intact mice from experimental colitis, but not NOD2or RICK-deficient mice. ${ }^{6,14,18}$ Collectively, these data support the idea that MDP activation of NOD2 downregulates innate immune responses to intestinal microflora and thus suggest that the absence of such regulation leads to increased susceptibility to CD.

In a previous study of the molecular mechanisms underlying NOD2 regulation of TLR response, we showed that MDP activation of NOD2 induces interferon regulatory factor 4 (IRF4) in human dendritic cells (DCs) and that such induction is necessary for the negative regulation of subsequent TLRinduced proinflammatory responses. ${ }^{14}$ In this study, we report that following NOD2 activation, IRF4 interacts with myeloid differentiation factor 88 (MyD88), TRAF6, and RICK and downregulates K63-linked polyubiquitinylation of RICK and TRAF6; this, in turn, disrupts NOD2- and TLR-MyD88induced NF- $\mathrm{KB}$ activation pathways, respectively. Thus, IRF4 function initiated by NOD2 activation provides new insight into how NOD2 influences colitis and why loss-of-function CARD15 polymorphisms serve as a risk factor in CD.

\section{RESULTS}

Physical interactions between IRF4 and MyD88, RICK, and TRAF6 in human DCs

In a previous study ${ }^{14}$ we showed that NOD2 prestimulation of human DCs results in inhibition of subsequent TLR-induced cytokine responses and that such inhibition requires the upregulation of IRF4. As for the mechanism of this IRF4 effect, we found in overexpression studies that NOD2 downregulation of TLR-mediated NF- $\mathrm{KB}$ activation is associated with IRF4 interaction with MyD88, RICK, and TRAF6; thus, it appeared that IRF4 was interfering with TLR signaling in some manner.

In this study, we addressed the molecular basis of this NOD2-IRF4 inhibitory mechanism by first assessing NOD2induced IRF4 physical interaction with RICK, MyD88, and TRAF6 in human DCs under physiologic conditions. For this purpose, DCs derived from healthy control peripheral blood monocytes (obtained by culturing the latter in the presence of granulocyte-macrophage colony-stimulating factor (GM-CSF) and interleukin (IL)-4) were prestimulated with MDP for $24 \mathrm{~h}$ and then restimulated with MDP or lipopolysaccharide (LPS). As shown in Figure 1a, MDP prestimulation (designated in
Figure 1 as first stimulation) upregulated endogenous IRF4 expression in human DCs without altering their constitutive expression of RICK, MyD88, or TRAF6.

Accompanying studies in which immunoprecipitation (IP) followed by immunoblotting (IB) was performed to assess signaling component interactions revealed that MDP prestimulation enhanced IRF4 binding to RICK, especially when it was accompanied by MDP restimulation. In addition, IRF4 binding to TRAF6 was barely seen in unstimulated cells or cells stimulated with MDP or LPS without prestimulation, but was clearly evident in cells prestimulated with MDP, especially when such prestimulation was followed by restimulation with MDP or LPS. Finally, IRF4 binding to MyD88 was increased in cells prestimulated with MDP and restimulated with LPS.

To verify the above IP-IB studies of interactions between IRF4 and various binding molecules in cells subjected to MDP stimulation, we employed the Duolink assay that allows fluorescent visualization of interacting proteins. ${ }^{19}$ As shown in Figure 1b, an IRF4-RICK complex was generated in both cells prestimulated with MDP and restimulated with MDP or LPS. In addition, IRF4-TRAF6 and IRF4-MyD88 complexes were generated in cells prestimulated with MDP and restimulated with either MDP or LPS, particularly the latter. Thus, both the IP-IB and Duolink assays reveal enhanced interactions between IRF4 and RICK, MyD88, and TRAF6 in human primary DCs prestimulated with MDP.

As also shown in Figure 1a, MDP prestimulation of cells was associated with reduced expression of phospho-IкB $\alpha$ and degradation of $I \kappa B \alpha$. Thus, the enhanced interaction between IRF4, RICK, and MyD88 in MDP-prestimulated human DCs is associated with downregulation of the NF- $\kappa \mathrm{B}$ signaling pathway.

IRF4 with mutations at two known phosphorylation sites is capable of both RICK-IRF4 interaction and IRF4 negative regulation

As RICK is a serine-threonine kinase, NOD2 activation of RICK could be regulating IRF4 function by enhancing RICK phosphorylation of IRF4. To investigate this possibility, we first determined the ability of RICK mutated at K47 (K47A) and/or S176 (S176A), sites previously shown to be necessary for RICK kinase activity. ${ }^{20,21}$ As shown in Figure 2a, binding of IRF4 to RICK (in proportion to RICK expression) was observed in 293 cells after the transfection of FLAG-tagged IRF4 along with RICK mutants lacking kinase activity.

In related studies, we determined the ability of IRF4 mutated at both S447 (S447A) and S448 (S448A), the potential serine phosphorylation sites previously shown to be necessary for IRF4 function in relation to IL-21 regulation, to bind to RICK. ${ }^{22}$ As shown in Figure 2b, both wild-type (WT) IRF4 and mutated IRF4 bound to RICK in 293 cells overexpressing V5-tagged RICK and FLAG-tagged IRF4. Thus, phosphorylation of IRF4 at these two potential phosphorylation sites was not required for the interaction between RICK and IRF4.

Finally, to assess the capacity of IRF4 lacking two serine phosphorylation sites to regulate NF- $\kappa B$ activity, we transfected 
a

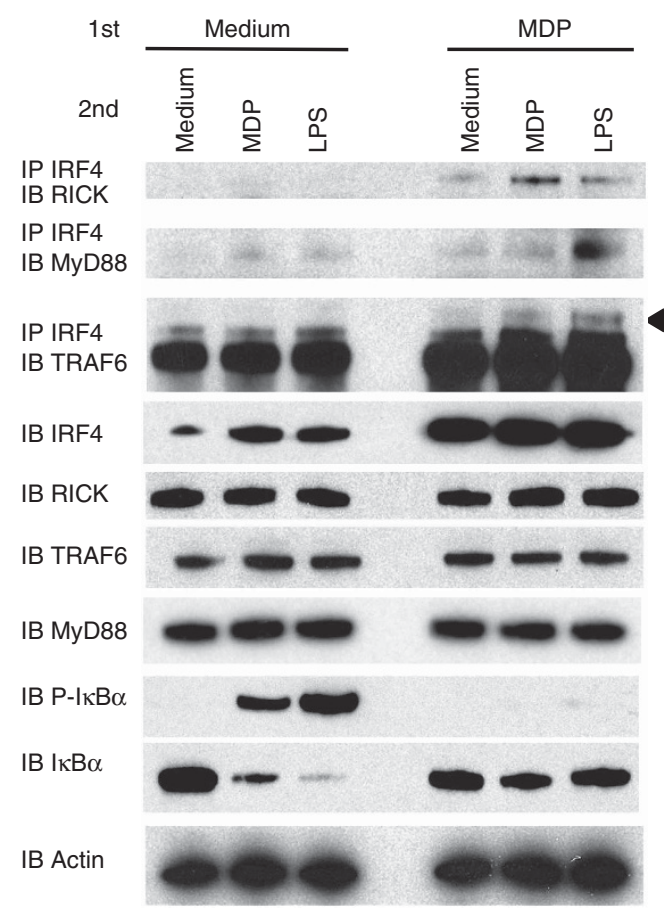

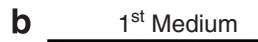
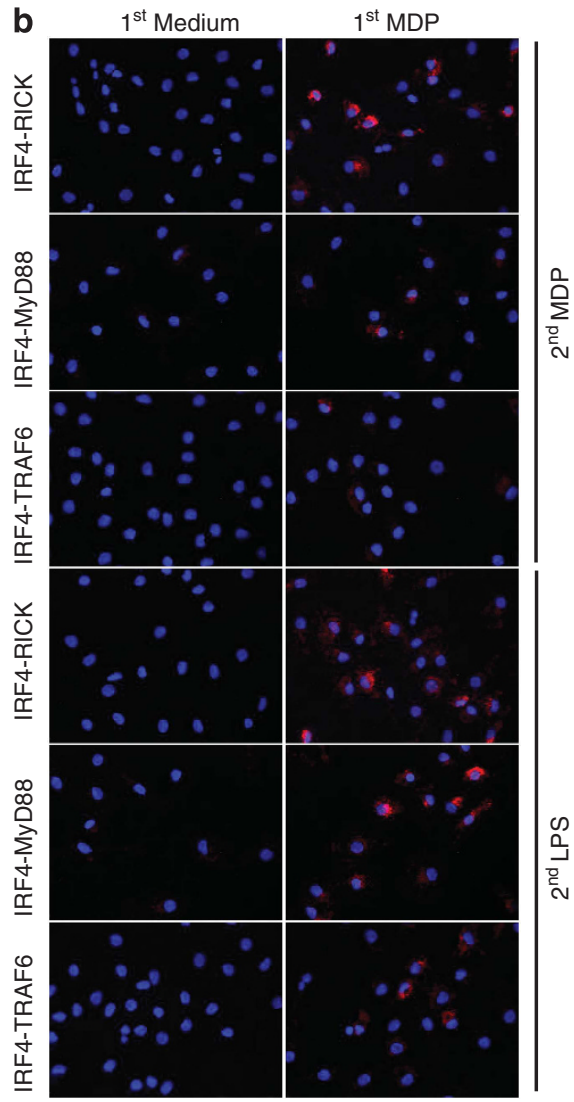

Figure 1 IRF4 expression induced by MDP activation of NOD2 and interaction between IRF4 and RICK, TRAF6, and MyD88. (a) Monocytes isolated from healthy individuals were cultured in the presence of GM-CSF and IL-4 for 6 days to generate monocyte-derived dendritic cells (DCs). DCs $\left(1 \times 10^{6}\right.$

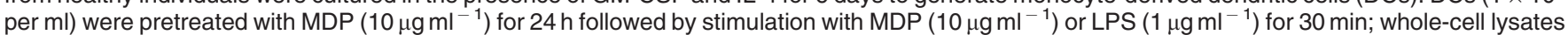
were subjected to immunoprecipitation (IP) with the indicated antibody (Ab) followed by immunoblotting (IB) with the indicated Ab. The arrow shows TRAF6-specific bands. (b) Human DCs were treated as described in (a). Interaction between IRF4 and RICK, TRAF6, and MyD88 was visualized in immunofluorescence microscopy by the Duolink assay. Original magnification $\times 800$. Results shown are representative of at least two experiments. GM-CSF, granulocyte-macrophage colony-stimulating factor; IL-4, interleukin-4; IRF4, interferon regulatory factor 4; LPS, lipopolysaccharide; MDP, muramyl dipeptide; MyD88, myeloid differentiation factor 88; NOD2, nucleotide-binding oligomerization domain 2; RICK, receptor interacting serine-threonine kinase; TRAF6, tumor necrosis factor receptor associated factor 6 .

plasmids expressing either WT or mutated IRF4 into HT-29 cells (i.e., nonhematopoietic cells not likely to express endogenous IRF4) ${ }^{23}$ cotransfected with a RICK-expressing plasmid as well as an NF- $\mathrm{KB}$ luciferase reporter-expressing plasmid. As shown in Figure 2c, transfection with mutated IRF4 led to a significant reduction in RICK activation of NF- $\kappa B$ as compared with that of WT IRF4. In complementary studies to address the same question in TLR-stimulated cells, we transfected either WT or mutated IRF4 into human THP1 cells that lack expression of IRF4 either before or after stimulation with MDP, as shown in our previous study, ${ }^{14}$ and then cultured the cells with TLR2 ligand $\left(\mathrm{Pam}_{3} \mathrm{CSK} 4, \mathrm{PAM}\right)$ or TLR4 ligand (LPS) to determine their capacity to produce NF- $\mathrm{KB}$-dependent cytokines. As shown in Figure 2d, transfection of mutated IRF4 into THP1 cells led to a significant decrease in TLR2 induction of IL-12p40 as compared with that of WT IRF4. Thus, these data provide evidence that loss of potential serine phosphorylation sites enhances increased IRF4-mediated negative regulation of NF- $\kappa \mathrm{B}$ activation and its downstream effect on cytokine production.
IRF4 regulates Lys63-linked polyubiquitination of RICK TLR and NOD-like receptor (NLR) activation of NF- $\kappa B$ requires K63 polyubiquitination of signaling components such as RICK and TRAF6. ${ }^{5-7,24,25}$ It was therefore possible that IRF4 expression induced by NOD2 activation inhibits NF- $\kappa B$ activation by downregulation of such polyubiquitination. To address this question, we first investigated the effect of IRF4 on RICK polyubiquitination in 293 cells transfected with plasmids expressing HA-tagged ubiquitin (Ub), V5-tagged RICK, and FLAG-tagged IRF4 and then lysed to obtain cell lysates that were subjected to IP with anti-HA antibody $(\mathrm{Ab})$ and $\mathrm{IB}$ with anti-V5 Ab.

As shown in Figure 3a, polyubiquitination of RICK was seen in 293 cells expressing HA-tagged WT Ub and V5-tagged RICK alone but not in cells also expressing IRF4. Consistent with previous studies showing that the E3 ligases, TRAF6 and cellular inhibitors of apoptosis proteins (cIAPs), induce K63-linked polyubiquitination of RICK, ${ }^{6,26}$ co-transfection of plasmids expressing TRAF6 or cIAP1 or cIAP2 enhanced polyubiquitination of RICK; however, such enhanced 
a

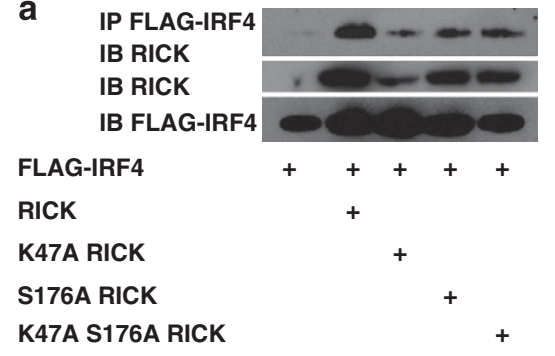

b IP V5-RICK

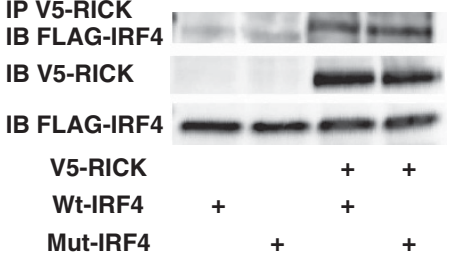

C

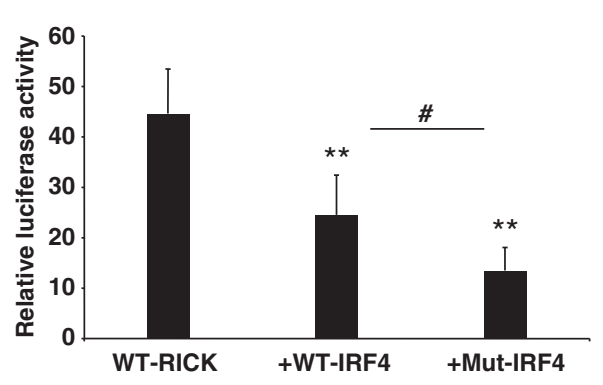

d
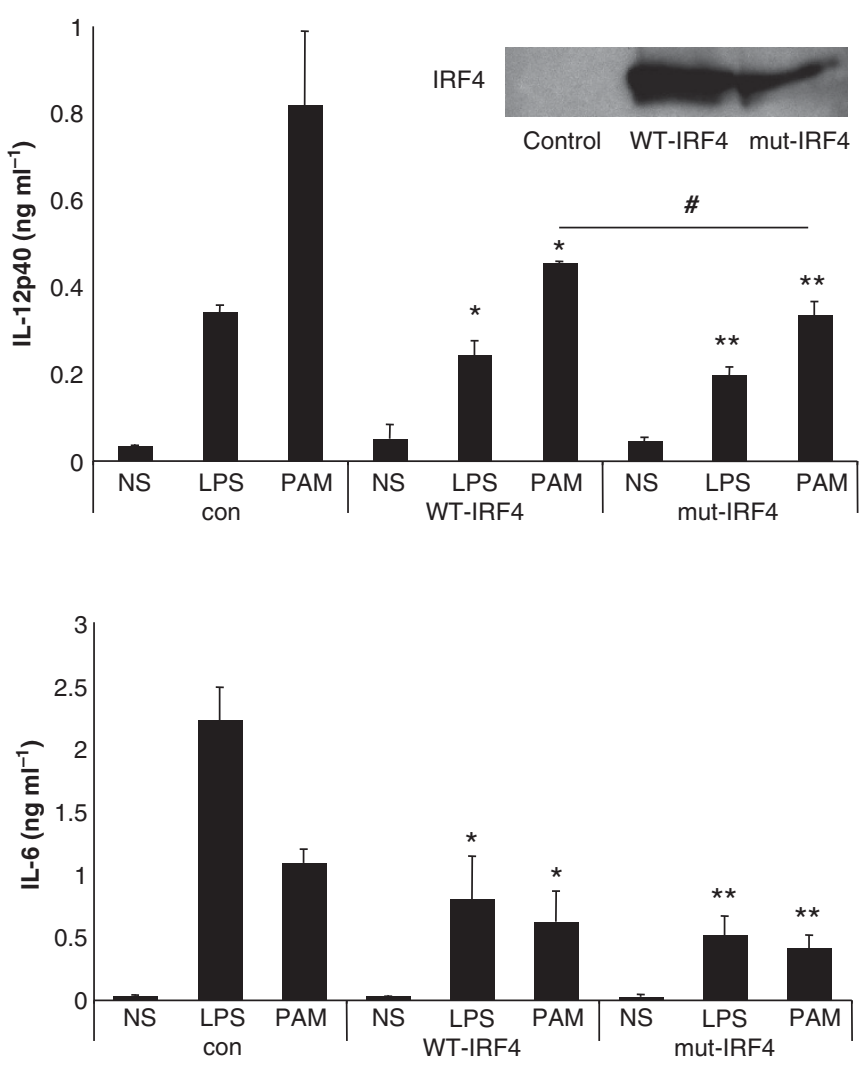

Figure 2 Phosphorylation of IRF4 is not necessary for its negative regulatory activity. (a) The 293 cells were transfected with vectors expressing FLAG-tagged IRF4 and several forms of mutated RICK. Whole-cell lysates were isolated $48 \mathrm{~h}$ after the transfection and were subjected to immunoprecipitation (IP) with the indicated antibody (Ab) followed by immunoblotting (IB) with the indicated Ab. (b) 293 cells were transfected with vectors expressing either wild type-IRF4 (Wt-IRF4) or Mutated-IRF4 (Mut-IRF4) in which serine residues at 447 and 448 are replaced with alanine; whole-cell lysates were isolated $48 \mathrm{~h}$ after transfection and were subjected to IP with anti-V5 Ab followed by IB with the indicated Ab. (c) HT29 cells were transfected with either empty control vector, Wt-IRF4, or Mut-IRF4-expressing vector together with RICK-expressing vector, NF- $\mathrm{BB}$ luciferase reporter vector, and $\mathrm{pSV}$ - $\beta$-galactosidase vector. The cells were then cultured for $24 \mathrm{~h}$ after which relative luciferase activity values were calculated. Results are expressed as mean \pm s.d. ${ }^{* *} P<0.01$ as compared with cells transfected with RICK vector alone. ${ }^{\#} P<0.01$. (d) THP1 cells were transfected with either empty control (con) vector, wt-IRF4, or mut-IRF4 vector. The cells were then stimulated with LPS or Pam ${ }_{3} \mathrm{CSK} 4$ (PAM) for $24 \mathrm{~h}$ after which culture fluids were analyzed for cytokine concentration by ELISA. Results were expressed as mean \pm s.d. ${ }^{\star} P<0.05$ and ${ }^{\star \star} P<0.01$ as compared with control vector. ${ }^{\#} P<0.01$. Results shown are representative of at least two experiments. ELISA, enzyme-linked immunosorbent assay; IRF4, interferon regulatory factor 4; LPS, lipopolysaccharide; NF- $\kappa B$, nuclear factor- $\kappa \mathrm{B}$; NS, no stimulation; RICK, receptor interacting serine-threonine kinase.

polyubiquitination of RICK was also markedly reduced in cells expressing IRF4 (Figure 3a,b). In further studies to determine the linkage type of poly-Ub chains on RICK, 293 cells were transfected with plasmids expressing mutant forms of Ub that specifically ubiquitinate via either K48 or K63 linkages. As shown in Figure 3a (middle and right panels), 293 cells expressing IRF4 exhibited reduced K63-linked polyubiquitination of RICK, even in cells cotransfected with TRAF6 that expressed increased amounts of K63-polyubiquitinated RICK; however, IRF4 expression had little or no effect on the marginal amount of K48-linked polyubiquitination observed, even in the presence of TRAF6.

As shown in Figure $\mathbf{3 b}$, enhanced K63-linked polyubiquitination of RICK induced by cIAP1 or cIAP2 (but not K48linked polyubiquitination) was also inhibited in cells expressing IRF4, whereas K63-linked polyubiquitination of RICK by ITCH, another E3 ligase known to induce such polyubiquitination, ${ }^{25}$ was not reduced in cells expressing IRF4 (data not shown). In addition, as shown in Figure 3c, reduced RICK polyubiquitination was seen in cells expressing either WT IRF4 or mutated IRF4 lacking its phosphorylation site, indicating that the inhibitory effect of IRF4 was not dependent on its phosphorylation status. Finally, as shown in Figure 3d, polyubiquitination of RICK induced by $30 \mathrm{~min}$ of stimulation with MDP in 293 cells stably expressing NOD2 was barely seen upon cotransfection of IRF4; thus, the inhibitory effect of IRF4 also applies to RICK activated by NOD2.

In related studies we identified RICK domains that interact with IRF4 and that are necessary for IRF4 inhibition of polyubiquitination. RICK is composed of a kinase domain, an intermediate domain, and a CARD domain. We therefore transfected plasmids expressing V5-tagged RICK mutants lacking each of these domains as well as RICK fragments composed of each of these domains into 293 cells cotransfected 


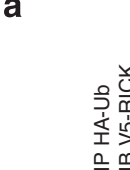

IB FLAG-IRF4

IB V5-RICK

IB TRAF6

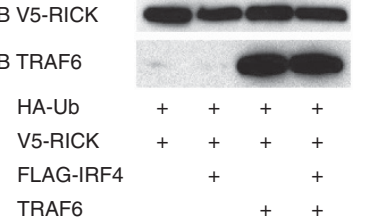

TRAF6
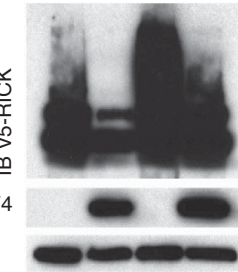

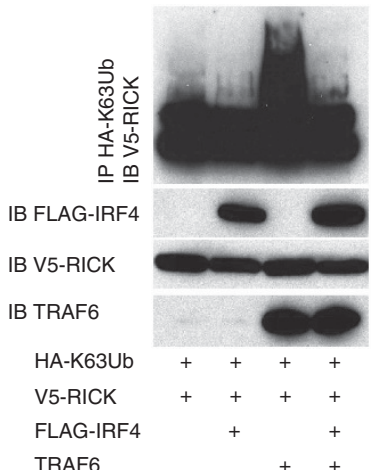

TRAF6

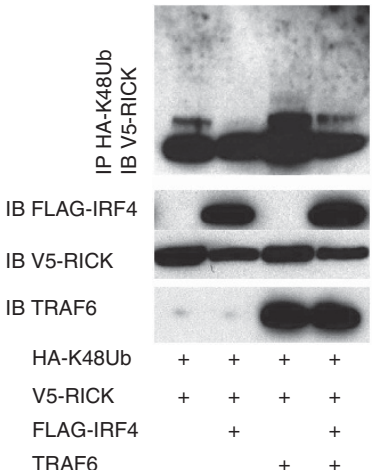

C

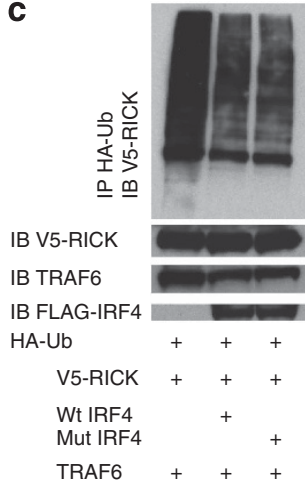

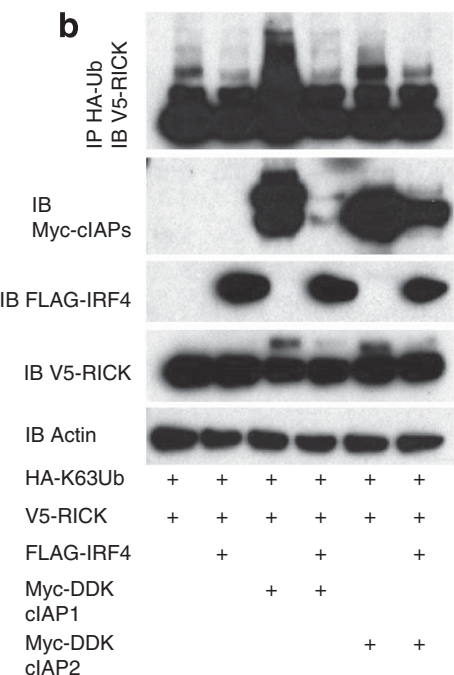
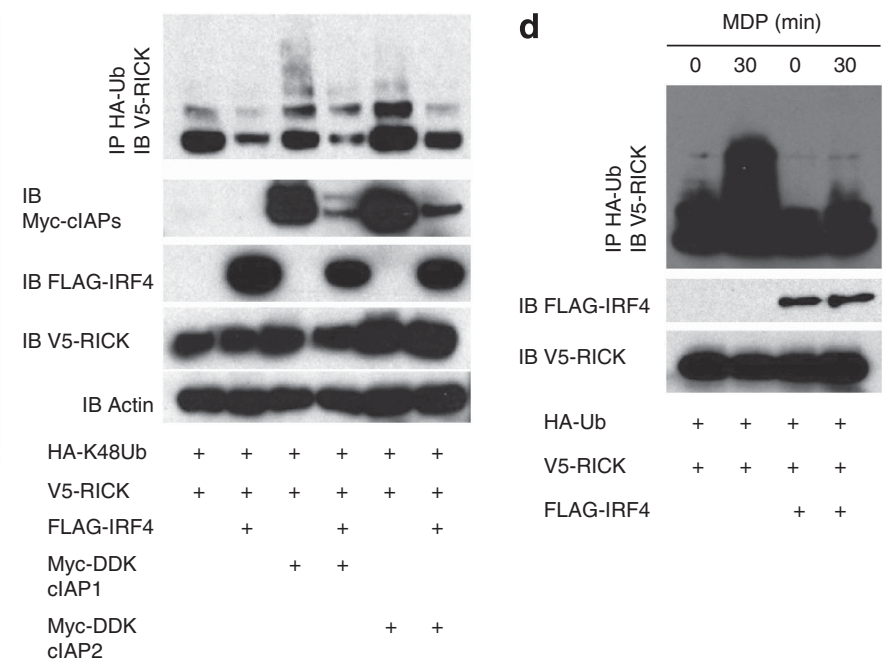

Figure 3 IRF4 regulates polyubiquitination of RICK. (a) The 293 cells $\left(1 \times 10^{6}\right.$ per 6 -well plate) were transfected with vectors $(1 \mu \mathrm{g})$ expressing HA-tagged wild-type ubiquitin (Ub), K48 Ub, K63 Ub, FLAG-tagged IRF4, V5-tagged RICK, and TRAF6; whole-cell lysates were prepared $48 \mathrm{~h}$ after the transfection and subjected to immunoprecipitation (IP) with the indicated antibody (Ab) followed by immunoblotting (IB) with the indicated Ab. (b) The 293 cells $\left(1 \times 10^{6}\right.$ per 6 -well plate) were transfected with vectors $(1 \mu \mathrm{g})$ expressing FLAG-tagged IRF4, Myc-DDK-tagged clAP1, Myc-DDK-tagged clAP2, V5-tagged RICK, and HA-tagged K48 or K63 Ub; whole-cell lysates were subjected to IP with the indicated Ab followed by IB with the indicated Ab. (c) The 293 cells $\left(1 \times 10^{6}\right.$ per 6 -well plate) were transfected with vectors $(1 \mu \mathrm{g})$ expressing HA-tagged wild-type Ub, V5-tagged RICK, TRAF6, FLAG-tagged IRF4, and FLAG-tagged mutated IRF4 (Mut IRF4) in which serine residues at 447 and 448 are replaced with alanine; whole-cell lysates were prepared $48 \mathrm{~h}$ after the transfection and subjected to IP with the indicated Ab followed by IB with the indicated Ab. (d) The 293 cells stably expressing NOD2 $\left(1 \times 10^{6}\right.$ per 6-well plate) were transfected with vectors $(1 \mu \mathrm{g})$ expressing HA-tagged wild-type Ub, FLAG-tagged IRF4, and V5-tagged RICK. At $48 \mathrm{~h}$ after the transfection, cells were stimulated with MDP $\left(10 \mu \mathrm{g} \mathrm{ml}^{-1}\right)$ for $30 \mathrm{~min}$; whole-cell lysates were subjected to IP with the indicated Ab followed by IB with the indicated $\mathrm{Ab}$. Results shown are representative of two experiments. IRF4, interferon regulatory factor 4; MDP, muramyl dipeptide; NOD2, nucleotidebinding oligomerization domain 2; RICK, receptor interacting serine-threonine kinase; TRAF6, tumor necrosis factor receptor associated factor 6.

with plasmids expressing FLAG-IRF4. As shown in Figure 4a, the interaction between RICK and IRF4 (defined by IP-IB studies) required the presence of the kinase and/or the intermediate RICK domains, as the interaction could be detected in 293 cells overexpressing kinase and intermediate domain RICK mutants but not in cells expressing the CARD domain mutant. Thus, the interaction between RICK and IRF4 was mediated by the binding of IRF4 to either the kinase or the intermediate domain but not to the CARD domain. With this information in hand, we next conducted ubiquitination studies of RICK domains in 293 cells transfected with plasmids expressing V5-RICK mutants or RICK fragments and cotransfected with plasmids expressing HA-Ub in the absence of IRF4 cotransfection. In previous studies, Hasegawa et al. ${ }^{7}$ showed that polyubiquitination of RICK at K209 in the kinase domain is essential for RICK-mediated NF- $\mathrm{KB}$ activation. As shown in
Figure 4b, consistent with this finding, 293 cells overexpressing mutant RICK lacking the kinase domain exhibited grossly defective polyubiquitination, whereas those overexpressing RICK mutants lacking the intermediate or CARD domains still exhibited polyubiquitination, although less than that of WT RICK; in addition, as also shown in Figure $\mathbf{4 b}$, whereas a kinase domain fragment of RICK was polyubiquitinated, an intermediate domain fragment of RICK was not. Thus, these studies demonstrate that the IRF4 inhibition of RICK polyubiquitination shown above involves polyubiquitination of the kinase domain of RICK, and this inhibition is facilitated by IRF4 binding to the kinase and/or intermediate domains of RICK.

\section{IRF4 regulates polyubiquitination of TRAF6}

Stimulation of the TLRs leads to the recruitment of MyD88 and TRAF6 to the receptor complex. ${ }^{27}$ This process activates 
a

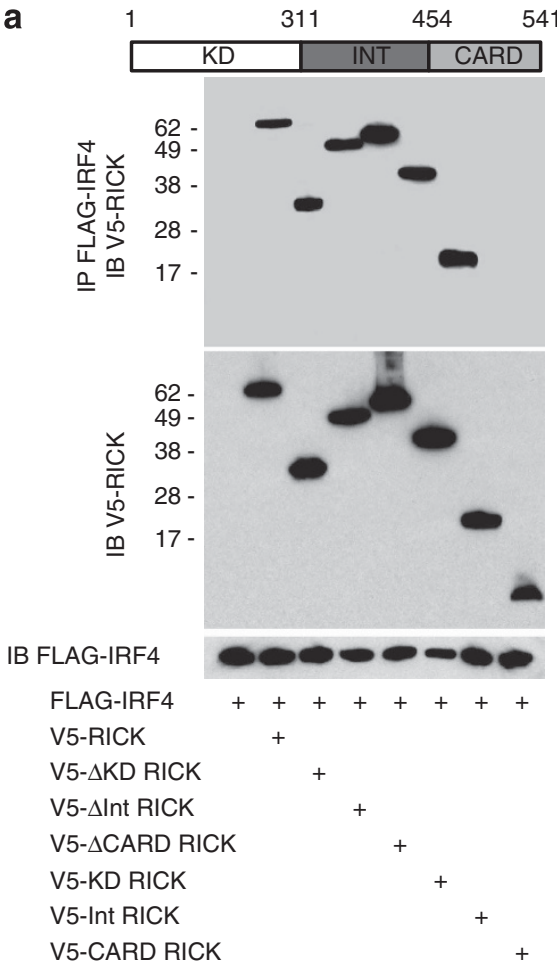

b

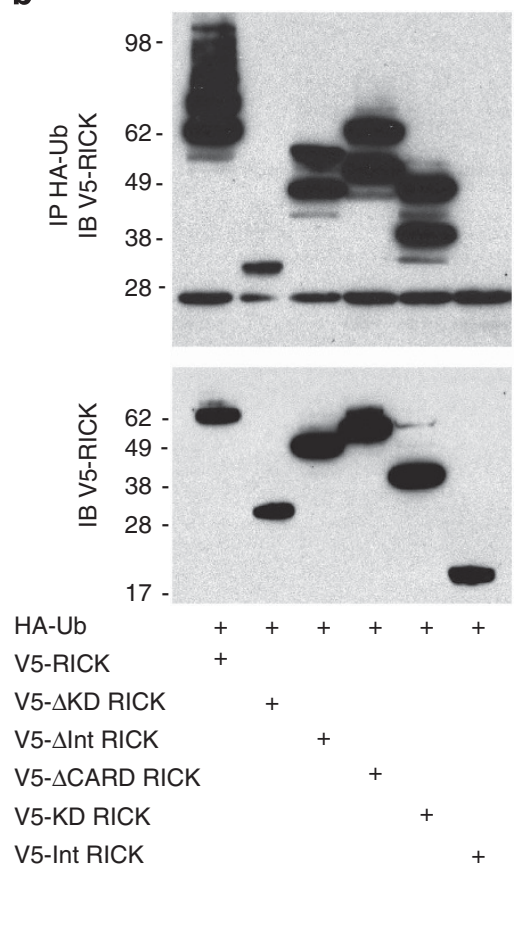

Figure 4 IRF4 inhibition of RICK polyubiquitination is facilitated by IRF4 binding to the kinase and/or intermediate domains of RICK. (a) The 293 cells were transfected with vectors expressing FLAG-tagged IRF4 and various forms of V5-tagged mutated RICK; whole-cell lysates were isolated $48 \mathrm{~h}$ after the transfection and were then subjected to immunoprecipitation (IP) with the indicated antibody (Ab) followed by immunoblotting (IB) with the indicated Ab. (b) The 293 cells were transfected with vectors expressing various forms of V5-tagged mutated RICK and HA-tagged ubiquitin (Ub); whole-cell lysates were isolated $48 \mathrm{~h}$ after the transfection and were subjected to IP with the indicated Ab followed by IB with the indicated Ab. Results shown are representative of two experiments. IRF4, interferon regulatory factor 4; RICK, receptor interacting serine-threonine kinase.

TRAF6 E3 ligase activity and TRAF6-mediated K63-linked polyubiquitination of downstream target proteins as well as TRAF6 itself. $^{24}$ As IRF4 binds to TRAF6 following MDP prestimulation, it seemed possible that, as in the case of RICK polyubiquitination, TRAF6 polyubiquitination was regulated by IRF4. To address this question, 293 cells were transfected with HA-tagged TRAF6, His-tagged Ub, and FLAG-tagged IRF4 and subjected to IP-IB studies as described above. As shown in Figure 5a,b, cells expressing WT or mutated IRF4 exhibited greatly reduced polyubiquitination of TRAF6 in the presence or absence of RICK. In addition, as shown in Figure 5c, robust TRAF6 polyubiquitination induced by $30 \mathrm{~min}$ of stimulation with LPS in 293 cells stably expressing TLR4, MD2, and CD14 was abolished in cells also expressing IRF4.

Taken together, these studies strongly suggest that IRF4 negatively regulates polyubiquitination of RICK and TRAF6 either in cells in which target proteins are being overexpressed and are being activated by NLR or TLR ligands, respectively.

\section{Prestimulation of human DCs with MDP inhibits Lys63-linked polyubiquitination of RICK and TRAF6}

To verify the results of the above overexpression studies of IRF4 under physiologic conditions, we next investigated whether IRF4 expression induced by NOD2 inhibits K63-linked polyubiquitination of RICK and/or TRAF6 in human DCs.
To this end, cell lysates prepared from human DCs that were prestimulated with MDP for $24 \mathrm{~h}$ and then restimulated with MDP or LPS were subjected to IP with Abs specific to K63- or K48-ubiquitin and then IB with Abs specific for RICK or TRAF6.

As shown in Figure 6a, prestimulation of human DCs with MDP greatly reduced K63-linked but not K48-linked polyubiquitination of RICK upon secondary stimulation with MDP of human DCs. Similarly, prestimulation with MDP greatly reduced K63-linked but not K48-linked polyubiquitination of TRAF6 upon secondary stimulation with LPS. It should be noted that LPS stimulation did not induce K63-linked polyubiquitination of RICK, and MDP stimulation did not induce K63-linked polyubiquitination of TRAF6; thus, the possible effect of prestimulation of cells with MDP in these situations could not be evaluated.

To determine whether the negative effect of MDP prestimulation on K63-linked polyubiquitination was in fact due to IRF4, we next conducted studies of cells in which IRF4 was downregulated by IRF4-specific small interfering RNA (siRNA; Figure 6b). As shown in Figure 6c, whereas MDP prestimulation of cells again led to inhibition of MDP-stimulated RICK and LPS-stimulated TRAF6 K63-linked polyubiquitination, respectively, such inhibition was reversed in cells treated with IRF4 siRNA. These studies show that in 


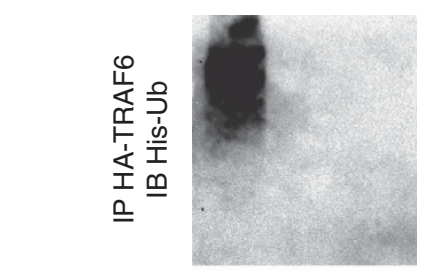

IB FLAG-IRF4

IB HA-TRAF6

$\begin{array}{llll}\text { His-Ub } & + & + & + \\ \text { HA-TRAF6 } & + & + & + \\ \text { Wt-IRF4 } & & + & \\ \text { Mut-IRF4 } & & & +\end{array}$

b

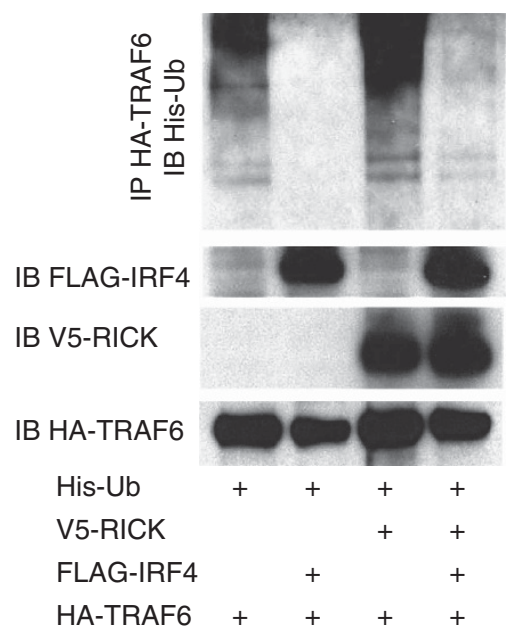

C

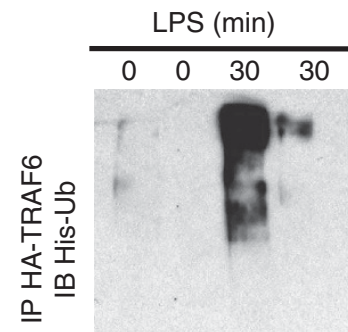

IB FLAG-IRF4

IB HA-TRAF6

His-Ub

HA-TRAF6

FLAG-IRF4

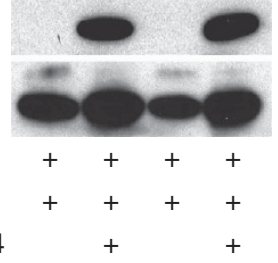

Figure 5 IRF4 regulates polyubiquitination of TRAF6. (a, b) The 293 cells $\left(1 \times 10^{6}\right.$ per 6 -well plate) were transfected with vectors $(1 \mu \mathrm{g})$ expressing His-tagged wild-type ubiquitin (Ub), FLAG-tagged IRF4, V5-tagged RICK, and HA-tagged TRAF6; whole-cell lysates were prepared 48 $\mathrm{h}$ after the transfection and subjected to immunoprecipitation (IP) with the indicated antibody (Ab) followed by immunoblotting (IB) with the indicated Ab. (c) The 293 cells stably expressing TLR4 $\left(1 \times 10^{6}\right.$ per 6 -well plate) were transfected with vectors $(1 \mu \mathrm{g})$ expressing His-tagged wild-type Ub, FLAG-tagged IRF4, and HA-tagged TRAF6. At $48 \mathrm{~h}$ after transfection, cells were stimulated with LPS $\left(1 \mu \mathrm{g} \mathrm{ml}^{-1}\right)$ for $30 \mathrm{~min}$; whole-cell lysates were subjected to IP with the indicated $\mathrm{Ab}$ followed by IB with the indicated Ab. Results shown are representative of two experiments. IRF4, interferon regulatory factor 4; LPS, lipopolysaccharide; RICK, receptor interacting serine-threonine kinase; TLR4, Toll-like receptor 4; TRAF6, tumor necrosis factor receptor associated factor 6.

physiologic human DCs, MDP activation of NOD2 and generation of IRF4 result in subsequent inhibition of MDPinduced RICK K63-linked polyubiquitination and LPS-induced TRAF6 K63-linked polyubiquitination. They thus fully corroborate the overexpression studies conducted with 293 cells.

As K63-polyubiquitination of RICK and TRAF6 have been shown to be necessary for TLR-NLR activation of NF- $\mathrm{KB},{ }^{5,24}$ we next asked whether downregulation of IRF4 expression by exposure to IRF4 siRNA also results in reversal of MDP prestimulation-induced inhibition of NF- $\mathrm{KB}$ activation. As shown in Figure 6d, culture of DCs in the presence of IRF4 siRNA did in fact abolish the capacity of MDP prestimulation to inhibit either MDP or LPS induction of NF- $\mathrm{KB}$ activation as assessed by expression of phospho-IкB $\alpha$, whereas control siRNA had no effect on inhibition. These results strongly suggest that IRF4 inhibition of RICK and TRAF6 K63-linked polyubiquitination results in downstream inhibition of NF- $\kappa \mathrm{B}$ activation.

\section{Administration of MDP protects mice from trinitrobenzene sulfonic acid (TNBS) colitis via regulation of K63-linked polyubiquitination of RICK and TRAF6}

The above results derived from in vitro studies of both 293 cells and human DCs suggest that MDP/NOD2-induced effects on K63-linked polyubiquitination in RICK and TRAF6 could explain our previous studies showing that MDP administration ameliorates experimental colitis. ${ }^{14}$ To address this possibility, we next determined the effect of MDP administration on K63-linked polyubiquitination in RICK and TRAF6 in mice with TNBS colitis. As shown in Figure $7 \mathbf{a}, \mathbf{b}$, and in the photomicrographs displayed in Supplementary Figure S1a online, systemic administration of MDP protected mice from the induction of TNBS colitis induced by intrarectal instillation of
TNBS, and such protection was accompanied by reduced nuclear translocation of NF- $\kappa B$ subunits (p65, p50, and c-Rel) in colonic lamina propria mononuclear cells (LPMCs). ${ }^{14}$

Importantly, several IRF4-related effects accompanied this MDP-mediated protection from colitis. Thus, as shown in Figure 7c, MDP administration led to markedly enhanced IRF4 expression in colonic tissue of MDP-treated mice and, as shown in Figure 7d, Duolink assays revealed that such administration also caused enhanced interaction between IRF4 and RICK, MyD88, or TRAF6 in such colonic tissue. Furthermore, as shown in Figure 7e, MDP administration led to reduced K63linked polyubiquitination of RICK and TRAF6 in colonic LPMCs that was unaccompanied by reduced K48-linked polyubiquitination. These in vivo studies are therefore consistent with the in vitro studies described above in showing that MDP-induced IRF4 expression and inhibition of K63-linked polyubiquitination of RICK and TRAF6 is associated with a downregulation of NF- $\kappa \mathrm{B}$; however, in this case, they show that this IRF4 effect is also associated with greatly reduced colonic inflammation.

\section{Prevention of TNBS colitis by administration of IRF4-expressing plasmids}

Whereas the above studies show that MDP-induced upregulation of IRF4 and the latter's effect on K63-linked ubiquitination of RICK and TRAF6 is associated with downregulation of NF- $\kappa B$ and colitis, it remained possible that MDP inhibition of $\mathrm{NF}-\kappa \mathrm{B}$ activation was occurring through another mechanism that does not involve IRF4. To address this possibility we conducted further in vivo studies in which we explored the possibility that exogenous IRF4 administration can directly prevent TNBS colitis, thereby by-passing MDP stimulation. 

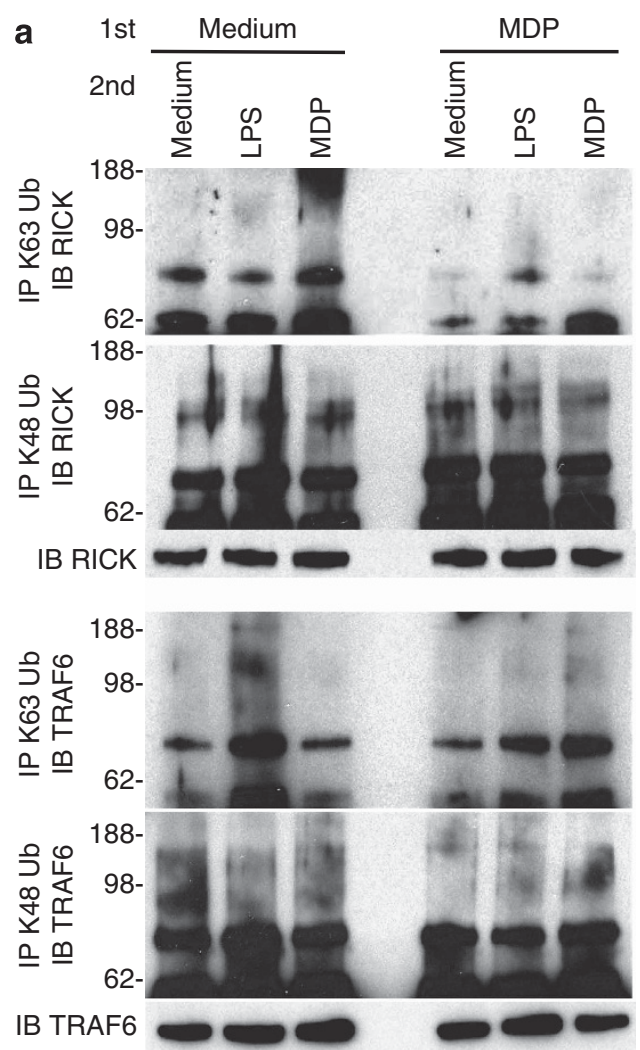

b

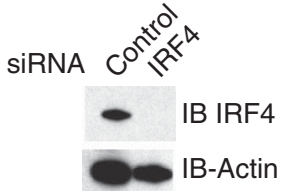

c
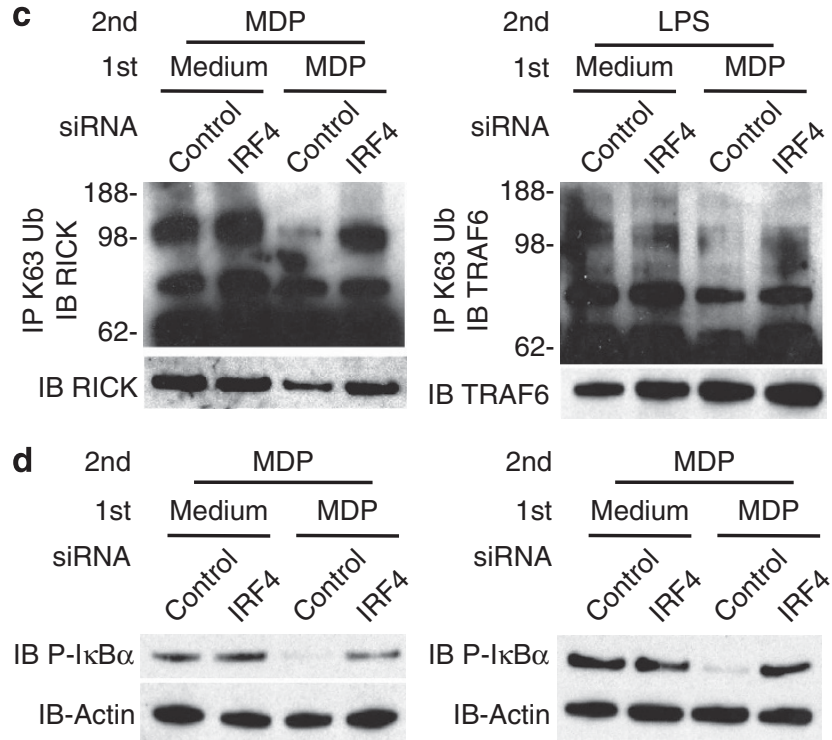

Figure 6 IRF4 expression induced by MDP activation of NOD2 inhibits K63-linked polyubiquitination of RICK and TRAF6. (a) Human monocyte-derived dendritic cells (DCs) were generated as described in Figure 1. DCs $\left(1 \times 10^{6}\right.$ per ml) were pretreated with MDP $\left(10 \mu \mathrm{g} \mathrm{ml}^{-1}\right)$ for $24 \mathrm{~h}$ followed by treatment with MG132 $\left(10 \mu \mathrm{g} \mathrm{ml}^{-1}\right)$ for $2 \mathrm{~h}$ and then were stimulated with MDP $\left(10 \mu \mathrm{g} \mathrm{ml}^{-1}\right)$ or LPS $\left(1 \mu \mathrm{g} \mathrm{ml}^{-1}\right)$ for 30 min. Whole-cell lysates were subjected to immunoprecipitation (IP) with the indicated antibody (Ab) followed by immunoblotting (IB) with the indicated Ab. (b) Human DCs were transfected with IRF4-specific siRNA or control siRNA $(100 \mathrm{~nm})$. At 1 day after transfection, DCs $\left(1 \times 10^{6}\right.$ per ml) were treated with MDP $\left(10 \mu \mathrm{g} \mathrm{ml}{ }^{-1}\right)$ for $24 \mathrm{~h}$ and then cell lysates were subjected to IB with the indicated Ab. (c) Human DCs were transfected with IRF4-specific siRNA or control siRNA (100 nM). At 1 day after transfection, DCs $\left(1 \times 10^{6}\right.$ per ml) were pretreated with MDP $\left(10 \mu \mathrm{g} \mathrm{ml}^{-1}\right)$ for $24 \mathrm{~h}$ followed by treatment with MG132 $\left(10 \mu \mathrm{g} \mathrm{ml} \mathrm{m}^{-1}\right)$ for $2 \mathrm{~h}$ and then were stimulated with MDP $\left(10 \mu \mathrm{g} \mathrm{ml}^{-1}\right)$ or LPS $\left(1 \mu \mathrm{g} \mathrm{ml}^{-1}\right)$ for $30 \mathrm{~min}$; whole-cell lysates were subjected to IP with the indicated Ab followed by IB with the indicated Ab. (d) Human DCs were transfected with IRF4-specific siRNA or control siRNA $(100 \mathrm{~nm})$. At 1 day after transfection, DCs $\left(1 \times 10^{6}\right.$ per ml) were pretreated with MDP $\left(10 \mu \mathrm{g} \mathrm{ml}^{-1}\right)$ for $24 \mathrm{~h}$ and then were stimulated with MDP $\left(10 \mu \mathrm{g} \mathrm{ml}^{-1}\right)$ or LPS $\left(1 \mu \mathrm{g} \mathrm{ml}^{-1}\right)$ for $30 \mathrm{~min}$; whole-cell lysates were subjected to IB with the indicated Ab. Results shown are representative of two experiments. IRF4, interferon regulatory factor 4; LPS, lipopolysaccharide; MDP, muramyl dipeptide; NOD2, nucleotide-binding oligomerization domain 2; RICK, receptor interacting serine-threonine kinase; siRNA, small interfering RNA; TRAF6, tumor necrosis factor receptor associated factor 6 .

Accordingly, we administered a FLAG-tagged IRF4 plasmid as well as a control plasmid encapsulated in a Hemagglutinin Virus of Japan-Envelope (HVJ-E) viral coat to mice by intrarectal instillation on each of the 2 days before and on the day of intrarectal challenge with TNBS. This method of inducing in vivo protein expression following plasmid administration had proven to be highly efficient in previous studies ${ }^{14}$ and, indeed, most of the colonic LPMCs expressing CD11b or CD11c were positive for the expression of FLAG-IRF4 in flow cytometric analysis 2 days after the induction of TNBS colitis (Supplementary Figure S2). As shown in Figure 8a and Supplementary Figure S1b, intrarectal administration of FLAG-tagged IRF4 plasmids prevented the development of TNBS colitis as assessed by body weight loss and colonic pathology analysis. Thus, exogenous IRF4 administration, like MDP administration, is an effective inhibitor of TNBS colitis.

In further studies, we investigated the interaction between the exogenously administered FLAG-tagged IRF4 and RICK,
TRAF6, and MyD88 in the colonic mucosa of the mice subjected to intrarectal TNBS challenge accompanied by IRF4 administration. To this end, whole protein extracts isolated from the colonic mucosa were subjected to IP with FLAG Ab and then IB with Abs specific for RICK, TRAF6, and MyD88. As shown in Figure 8b, administered FLAG-tagged IRF4 was bound to RICK, MyD88, and TRAF6. In a final set of studies we determined the effect of the exogenously administered IRF4 on NF- $\mathrm{KB}$ and MAP kinase expression as well as on downstream proinflammatory cytokine expression. As shown in Figure 8c,d, exogenously administered IRF4 led to markedly reduced expression of phospho-IкB $\alpha$, phospho-p38, and phospho-ERK, as well as reduced nuclear translocation of $\mathrm{NF}-\mathrm{KB}$ subunits in the colonic mucosa of mice treated with intrarectal administration of FLAG-tagged IRF4 plasmids as compared with those treated with control plasmid. In addition, evidence of reduced activation of NF- $\mathrm{KB}$ was associated with reduced pro-inflammatory cytokine responses by 
a
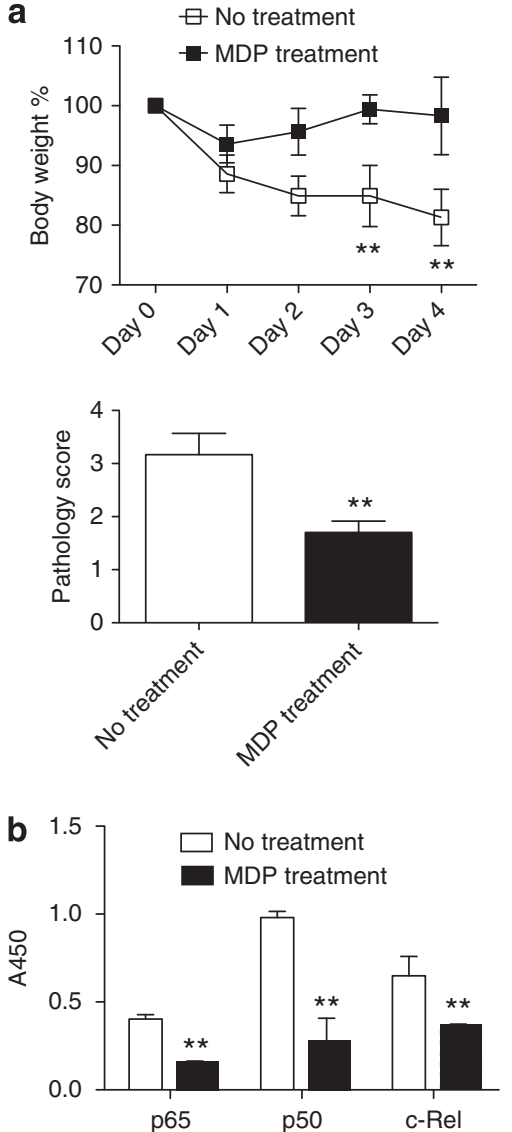

C
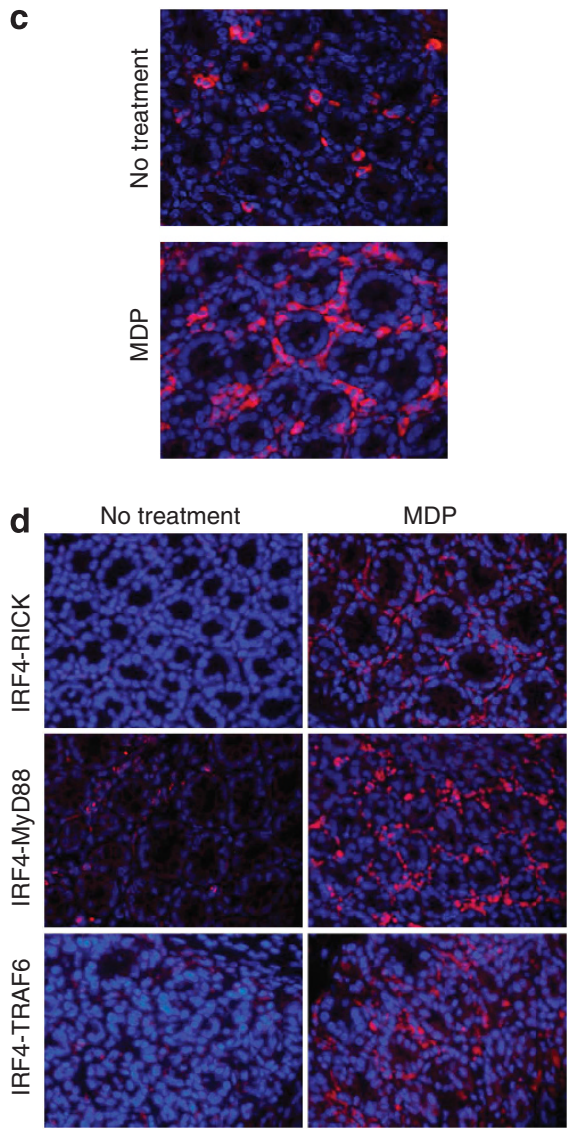

e
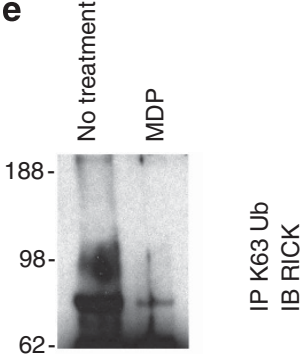

$188-$

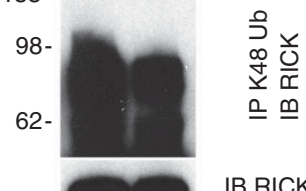

IB RICK

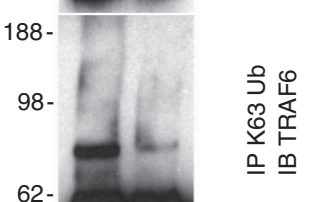

188

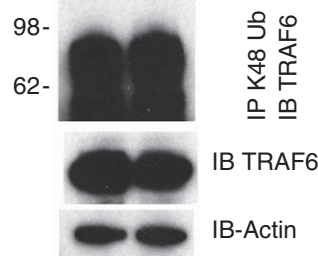

Figure 7 Administration of MDP protects mice from trinitrobenzene sulfonic acid (TNBS) colitis via regulation of K63-linked polyubiquitination of RICK and TRAF6. C57BL/10 mice were administered MDP on days $-2,-1$, and 0 and then challenged with intrarectal TNBS on day 0. (a, upper panel) Changes in body weight of mice not treated or treated with MDP $(n=10)$ and challenged with intrarectal administration of TNBS. (a, lower panel) Pathology scores of mice not treated or treated with MDP on day 4. Results were expressed as means $\pm s$.d. ${ }^{\star \star} P<0.01$ as compared with untreated group. (b) NF-KB activation in colonic LPMCs in mice challenged with TNBS. Nuclear extracts were isolated from colonic LPMCs on day 1 and subjected to a Transfactor Binding Assay. Results are expressed as means \pm s.d. ${ }^{* \star} P<0.01$ as compared with untreated group. (c) IRF4 expression in the colonic tissue of mice challenged with TNBS on day 1. (d) Interaction between IRF4 and RICK, MyD88, and TRAF6 in the colonic tissue of TNBS-challenged mice on day 1. Molecular interactions were visualized by Duolink assay (red color). (e) K48- or K63-linked polyubiquitination of RICK and TRAF6 in colonic LPMCs of TNBS-challenged mice. Whole-cell lysates prepared from colonic LPMCs on day 1 were subjected to immunoprecipitation (IP) with the indicated antibody $(\mathrm{Ab})$ followed by immunoblotting (IB) with the indicated Ab. IRF4, interferon regulatory factor 4; LPMC, lamina propria mononuclear cell; MDP, muramyl dipeptide; MyD88, myeloid differentiation factor 88; NF- $\mathrm{KB}$, nuclear factor- $\mathrm{kB}$; RICK, receptor interacting serine-threonine kinase; TRAF6, tumor necrosis factor receptor associated factor 6.

colonic LPMCs. Thus, as shown in Figure 8e,f, colonic LPMCs produced markedly less IL-12p40 and interferon- $\gamma$ upon stimulation with MDP, $\mathrm{Pam}_{3} \mathrm{CSK} 4$ (PAM, a TLR2 ligand), LPS, flagellin (a TLR5 ligand), CPG (a TLR9 ligand), and anti-CD3 Ab.

Taken together, these data show that IRF4 administration mimics the effect of MDP administration and increased NOD2 signaling during induction of TNBS colitis as previously reported. ${ }^{14}$ As such, they show that prevention of TNBS colitis by MDP administration is most likely acting via the induction of IRF4.

\section{Treatment of TNBS colitis by administration of IRF4-expressing plasmids}

The striking effect of IRF4-expressing plasmid administration on TNBS colitis shown above suggested that such administration could have therapeutic value. To address this possibility, we determined whether administration of IRF4-expressing plasmid could reverse previously established TNBS colitis. Accordingly, mice were challenged with TNBS as described above but in this case they were administered FLAG-tagged IRF4 plasmid encapsulated in the HVJ-E vector 3 days after TNBS administration rather than at the time of TNBS administration. As shown in Figure 9a, expression of FLAG was again shown to be present in the colonic mucosa of mice following FLAG-tagged IRF4 plasmid administration. In addition, as shown in Figure 9b and Supplementary Figure S1c, such mice promptly regained body weight and exhibited improved microscopic inflammation scores. Moreover, as shown in Figure 9c-e, administration of IRF4 was followed by reduced nuclear translocation of NF- $\mathrm{KB}$ components in the colonic mucosa as well as greatly decreased production of proinflammatory cytokines by mesenteric lymph node cells upon stimulation with TLR ligands and anti-CD3 monoclonal 
a
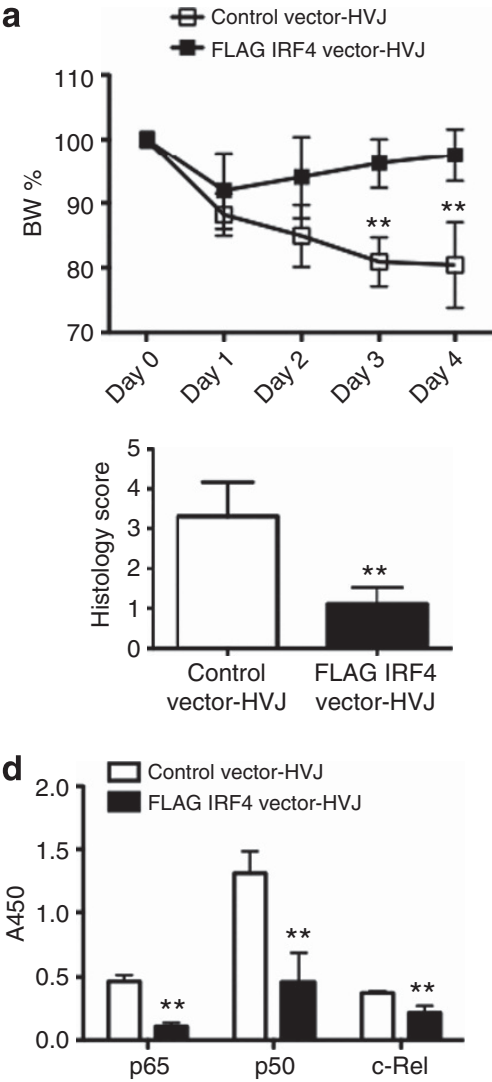

b
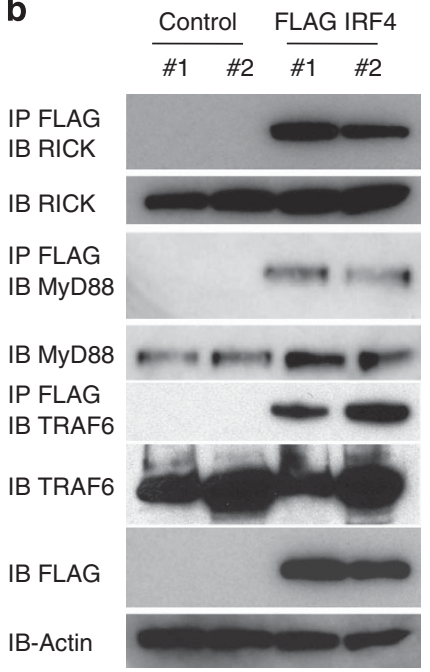

C
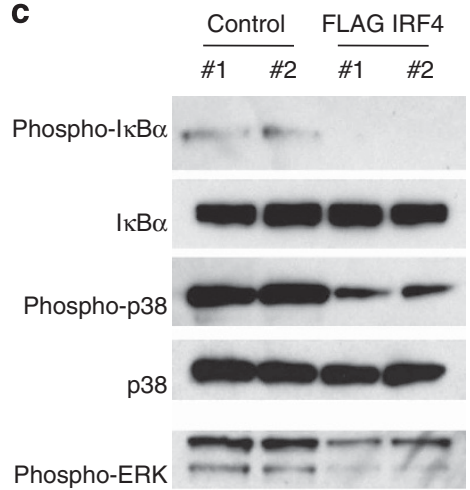

Phospho-ERK

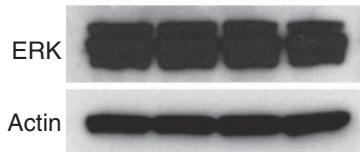

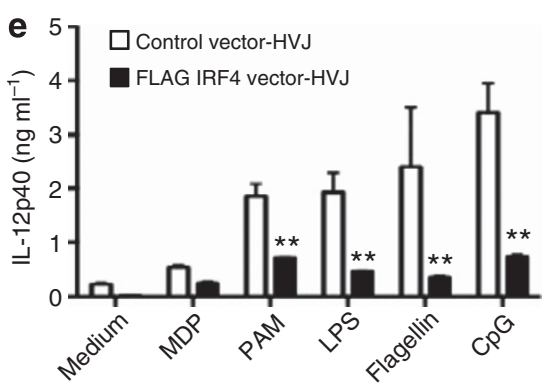

f

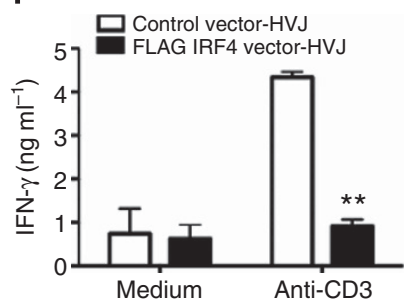

Figure 8 Prevention of trinitrobenzene sulfonic acid (TNBS) colitis by administration of an IRF4-expressing plasmid. C57BL/10 mice were administered HVJ-encapsulated FLAG-tagged IRF4 vector or control vector via the intrarectal route on days $-2,-1$, and 0 and then challenged with intrarectal TNBS on day 0. (a) Changes in body weight in mice $(n=5$; each group, top panel) and pathology scores of mice on day 4 . Results were expressed as means \pm s.d. ${ }^{* *} P<0.01$ as compared with control vector group. (b) Interaction between FLAG-tagged IRF4 and RICK, MyD88, and TRAF6 in the colonic tissue of mice. Whole protein lysates were prepared from the colon tissues of TNBS-challenged mice on day 2 and were subjected to immunoprecipitation (IP) with the indicated antibody (Ab) followed by immunoblotting (IB) with the indicated Ab. Two colon extracts from each group were used for the assay. (c) Activation of NF-кB and MAPK in the colon tissues of TNBS-challenged mice on day 2. Two colon extracts from each group were used for the immunoblotting. (d) NF-kB activation in colon tissues in mice challenged with TNBS. Nuclear extracts were isolated from colon tissues on day 2 and subjected to a Transfactor Binding Assay. Results were expressed as means \pm s.d. ${ }^{\star \star} P<0.01$ as compared with control vector group. (e, f) Production of IL-12p40 and interferon- $\gamma$ (IFN- $\gamma$ ) by colon LPMCs isolated from TNBS-challenged mice on day 4 . Colon LPMCs $\left(1 \times 10^{6}\right.$ per ml) were stimulated with MDP, PAM, LPS, Flagellin, and CpG for $24 \mathrm{~h}$ or with anti-CD3 Ab for $48 \mathrm{~h}$ after which culture fluids were assayed for IL-12p40 or IFN- $\gamma$ levels by enzymelinked immunosorbent assay (ELISA), as indicated. Results were expressed as means \pm s.d. ${ }^{* *} P<0.01$ as compared with group-administered control vector. Results shown are representative of two experiments. HVJ, hemagglutinin virus of Japan; IL, interleukin; IRF4, interferon regulatory factor 4; LPMC, lamina propria mononuclear cell; LPS, lipopolysaccharide; MAPK, mitogen-activated protein kinase; MDP, muramyl dipeptide; MyD88, myeloid differentiation factor 88 ; NF- $\kappa B$, nuclear factor- $\mathrm{kB}$; RICK, receptor interacting serine-threonine kinase; TRAF6, tumor necrosis factor receptor associated factor 6 .

$\mathrm{Ab}$. These data thus provide further proof that MDP activation of NOD2 and generation of IRF4 leads to downregulation of $\mathrm{NF}-\kappa \mathrm{B}$ activation and colonic inflammation. In addition, they suggest that IRF4 administration might have clinical value.

\section{DISCUSSION}

In a previous study, we demonstrated that MDP activation of NOD2 has downregulatory effects on multiple TLR signaling pathways. ${ }^{14}$ We now provide a mechanism for such downregulation by showing that MDP activation of NOD2 induces IRF4-mediated inhibition of molecular events essential to the activation of NF- $\mathrm{KB}$, namely K63-linked polyubiquitination of RICK and TRAF6. The chain of evidence supporting this conclusion consisted first of the fact that MDP prestimulation of human DCs leads to binding of RICK to IRF4 and the binding of IRF4 to TRAF6 and MyD88. As shown in overexpression studies conducted in a cell line and more importantly in physiologic human DCs, these interactions set the stage for MDP/NOD2-induced IRF4 inhibition of K63linked polyubiquitination of RICK and TRAF6; in addition, such inhibition could be linked to downregulation of NF- $\mathrm{KB}$ activation by the demonstration that siRNA downregulation of IRF4 reverses the negative effect by NOD2 signaling on K63linked polyubiquitination and NF- $\mathrm{KB}$ activation. These in vitro studies were then supported by in vivo studies showing that protection from the development of TNBS colitis by MDP administration is accompanied by greatly increased IRF4 expression and interaction with RICK, TRAF6, and MyD88, as well as inhibition of K63-linked polyubiquitination of RICK and TRAF6 in colonic cells and this, in turn, is associated with 
a

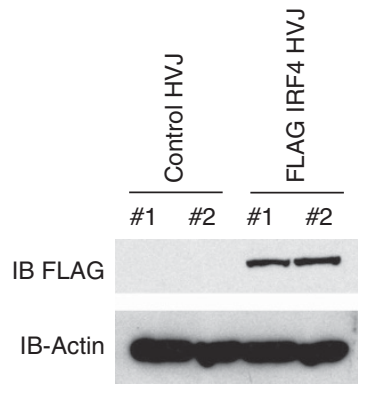

b
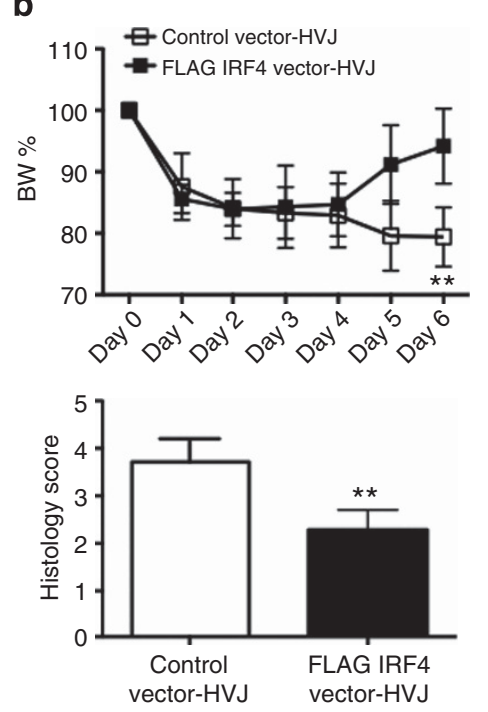

c

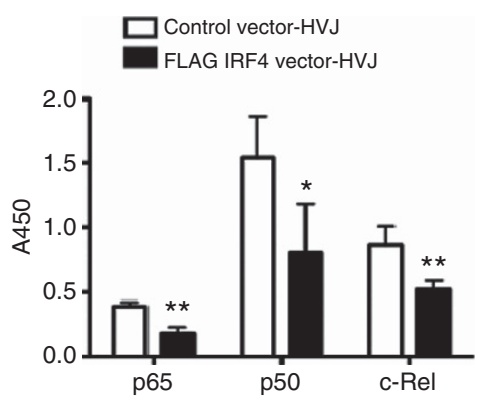

d
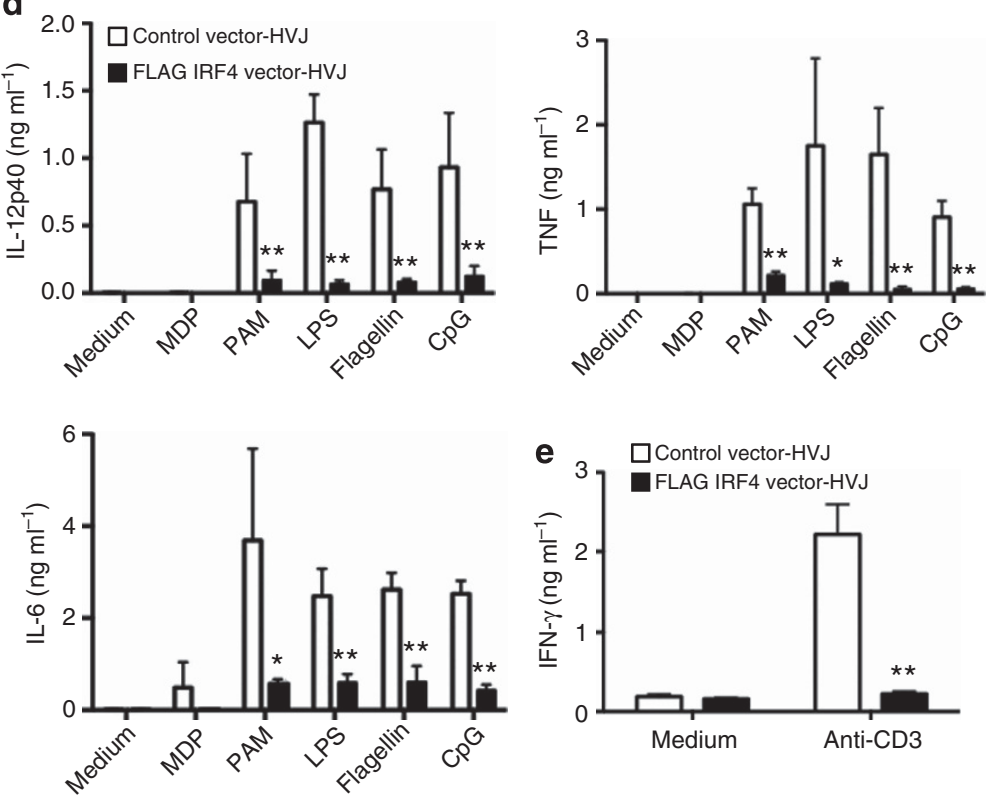

Figure 9 Treatment of trinitrobenzene sulfonic acid (TNBS) colitis by administration of an IRF4-expressing plasmid. C57BL/10 mice were administered HVJ-encapsulated FLAG-tagged IRF4 vector or control vector via the intrarectal route on day 3 after challenge with TNBS on day 0 . (a) Expression of FLAG-tagged IRF4 in the colonic tissues of mice. Whole protein lysates were prepared from the colonic tissues of TNBS-challenged mice on day 6 and were subjected to immunoblotting. Two colonic extracts from each group were used for immunoblotting. (b) Changes in body weight in mice ( $n=7$; each group, top panel) and pathology scores of mice on day 6 . Results were expressed as means \pm s.d. Results shown are a pool of two independent

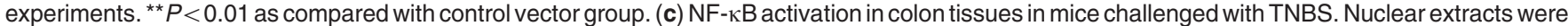
isolated from colon tissues on day 6 and subjected to Transfactor binding assay. Results were expressed as means \pm s.d. ${ }^{*} P<0.05$ and ${ }^{* *} P<0.01$ as compared with control vector group. (d, e) Production of IL-12p40, TNF, IL-6 and IFN- $\gamma$ by mesenteric lymph node (MLN) cells isolated from TNBSchallenged mice on day 6 . MLN cells $\left(2 \times 10^{6}\right.$ per $\left.\mathrm{ml}\right)$ were stimulated with MDP, PAM, LPS, Flagellin, and CpG for $24 \mathrm{~h}$ to determine the protein levels of IL-12p40, TNF, and IL-6. Cells were also stimulated with anti-CD3 antibody (Ab) for $48 \mathrm{~h}$ to determine the protein levels of IFN- $\gamma$. Results were expressed as means \pm s.d. ${ }^{*} P<0.05$ and ${ }^{* *} P<0.01$ as compared with control vector group. HVJ, hemagglutinin virus of Japan; IFN- $\gamma$, interferon- $\gamma$; IL, interleukin;

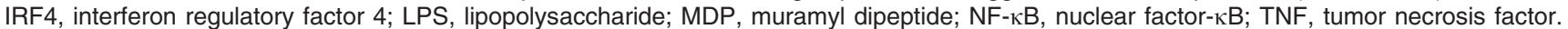

greatly reduced NF- $\kappa B$ activation. A final step in the chain of evidence consisted of studies showing that administration of an IRF4-expressing plasmid to mice both prevented TNBS colitis and reversed already established TNBS colitis. These latter studies established that MDP activation of NOD2 in vivo was in fact acting via IRF4 to inhibit NF- $\kappa$ B activity. Overall, these data provide a mechanistic explanation of how MDP-NOD2 stimulation of APCs negatively regulates inflammatory responses induced by TLR ligands and therefore explain how defective NOD2 function can lead to excessive TLR responses in the gut that contribute to the pathogenesis of $\mathrm{CD}$.

IRF4 has been shown in previous reports to function as a negative regulator of TLR signaling pathways in innate immune cells such as DCs. ${ }^{14,28,29}$ As for the mechanisms accounting for such negative regulation, Negishi et al $^{28}$ provided evidence that
IRF4 expression is upregulated by activation of TLRs and then competes with IRF5 for binding to and activation of MyD88. Although this mechanism of IRF4 inhibitory activity may be valid, it cannot be the only mechanism in play because we have shown previously as well in the present studies that MDP prestimulation acting through NOD2 inhibits LPS and other TLR ligand cytokine responses, including TLR responses that do not involve MyD88. ${ }^{14}$ It should be noted in this context that whereas NOD2 activation leads to RICK-IRF4 binding, LPS activation in the absence of MDP-induced NOD2 interaction does not have this effect (Watanabe, T., Asano, N., and Strober, W., unpublished observation). Thus, it is possible that NOD2 activation leads to RICK-IRF4 complexes that have inhibitory functions not shared by IRF4 alone. In any case, we have shown that systemic administration of MDP to mice 
challenged with TNBS to induce TNBS colitis leads to enhanced expression of IRF4 in colonic LPMCs and inhibition of inflammation due to reduced K63-linked polyubiquitination of TRAF6 and RICK in those cells. In addition, intrarectal administration of an IRF4-expressing plasmid to mice challenged with TNBS had a similar anti-inflammatory effect. Thus, our study disclosed a molecular mechanism by which NOD2-induced IRF4 inhibits intestinal inflammation driven, at least in part, by activation of TLR signaling pathways that are not regulated by TLR-induced IRF4.

In the above studies of TNBS colitis, the development of colitis was inhibited either by systemic injection of MDP or intrarectal administration of IRF4 complementary DNA. These treatments were parallel in that administration of MDP resulted in enhanced interaction between IRF4 and RICK, TRAF6, and MyD88, and administration of IRF4 complementary DNA led to interaction of IRF4 with the same signaling molecules; moreover, reduction of NF- $\kappa B$ activation was seen in both treatments. Thus, it is likely that MDP activation of NOD2 prevents experimental colitis via induction of IRF4 expression. It should be noted that this conclusion leads to the assumption that activation of TLRs with the ability to induce the expression of IRF4 have the potential to inhibit experimental colitis via the same deubiquitination mechanism described here for NOD2. However, previous reports show that generation of tolerogenic responses by LPS depends upon the induction of IRAK-M (IL-1 receptor-associated kinase M) rather than IRF4. ${ }^{14,29}$ Thus, tolerogenic responses induced by MDP activation of NOD2 appear to be intrinsically different from those induced by LPS activation of TLR4, and although LPS administration may suppress colitis because of induction of LPS tolerance, such suppression will not be mediated by the deubiquitinization function of IRF4.

Of interest, IRF4 may be exerting inhibitory effects by a mechanism similar to that described here in other instances of proinflammatory responses. This includes IRF4-mediated suppression of TLR-induced cytokine responses, leading to acute postischemic renal inflammation by IRF4 generated in renal DCs reacting to oxidative stress. ${ }^{30}$ and IRF4-mediated reduction of TLR-induced proinflammatory responses of liver plasmacytoid DCs by IRF4 generated in such cells by in vivo administration of MDP. ${ }^{13}$ However, additional studies will be necessary to verify this possibility.

IRF4 is expressed in most types of cells in the lymphoid system and may have cell- specific functions. ${ }^{31}$ This possibility is illustrated by the fact that, in contrast to the anti-inflammatory role of IRF4 in TNBS colitis and postischemic renal inflammation described above, it has a proinflammatory role in experimental $\mathrm{T}$ cell-mediated autoimmune disorders. ${ }^{31}$ The latter may be because of the fact that IRF4 has been shown to be a positive regulator of IL-17 and IL-21 responses in T cells and it increased responsiveness to IL-21 in B cells. ${ }^{22,31,32}$ Interestingly, there is evidence that this proinflammatory activity by IRF4 plays a critical role in a T cell-dependent colitis. Thus, Mudter et al. ${ }^{33,34}$ have shown that adoptive transfer of naive $\mathrm{CD} 4^{+} \mathrm{T}$ cells from WT mice, but not IRF4-deficient mice, to immune-deficient mice resulted in severe colitis associated with increased production of IL- 6 and T helper type 17 cytokines. In our studies, intrarectal delivery of a plasmid expressing FLAG-tagged IRF4 was shown to lead to marked expression of FLAG-tagged IRF4 in colonic APCs rather than in colonic $\mathrm{T}$ cells. Thus, our data support the idea that whereas IRF4 expression in T cells may promote the development of colitis, its expression in APCs has the contrary effect. In this context, it should also be noted that the HVJ-E delivery of IRF4-expressing plasmid could have led to IRF4 expression in nonmyeloid cells, and thus we cannot rule out the possibility that the ability of exogenous IRF4 expression to affect colitis in these studies could have been mediated by effects on one or another nonmyeloid cell.

As shown by the fact that intrarectal administration of NF- $\kappa B$ decoy oligonucleotides that blocks activation of NF- $\kappa B$ target genes prevents and treats TNBS or oxazolone colitis, the pathogenic immune responses underlying experimental colitis depend on NF- $\mathrm{KB}$ transcriptional activity. ${ }^{35,36}$ In recent years, evidence has accumulated that such NF-KB-mediated inflammation is tightly regulated by ubiquitination of various components of the NF- $\mathrm{KB}$ signaling cascade. This is well illustrated by the fact that the function of TRAF6, an essential component of the signaling cascade leading to NF- $\mathrm{KB}$ activation, is dependent on its K63-linked polyubiquitination status. Thus, deubiquitination of TRAF6 by the deubiquitinating enzyme, A20, leads to inhibition of TLRs-induced NF- $\kappa B$ activation, ${ }^{24,37}$ and A20 deficiency results in excessive NF- $\mathrm{\kappa B}$ activation associated with the development of autoimmune disease or colitis due to stimulation by TLR ligands derived from the intestinal microflora. ${ }^{24,38}$ In this study, we show that downregulation of K63linked polyubiquitination of TRAF6 resulting from an inhibitory interaction with IRF4 also leads to decreased NF- $\mathrm{KB}$ activation. The mechanism of such IRF4 inhibition is presently unknown. However, given the fact that TRAF6 is an E3-ligase and can therefore facilitate both the polyubiquitination of downstream signaling molecules and its own polyubiquitination, one possible mechanism is that IRF4 interferes with TRAF6 E3-ligase activity.

Despite the fact that CARD15 polymorphisms associated with $\mathrm{CD}$ are considered to be loss-of-function abnormalities that lead to decreased NOD2-mediated proinflammatory function, immune responses linked to $\mathrm{CD}$ are characterized by production of proinflammatory cytokines driven by excessive NF- $\kappa \mathrm{B}$ activation. ${ }^{39,40}$ One explanation of this paradox is that NOD2 signaling in epithelial cells is necessary for the elaboration of epithelial cell-derived cryptins that ordinarily control bacterial growth in the intestine; thus, loss of NOD2 function can lead to excessive bacterial growth that gives rise to colitis. ${ }^{41,42}$ It should be noted, however, that chronic NOD2 activation has been shown to mediate downregulation of innate TLRs responses to microbial antigens in the intestine, a phenomenon known as cross-tolerance. ${ }^{14-17}$ Therefore, an alternative explanation of how CARD15 polymorphisms and loss of NOD2 function leads to intestinal disease is that NOD2 regulates innate responses to intestinal microflora by suppressing TLR responses in the healthy intestinal mucosa, whereas in the diseased CD intestinal mucosa lack of NOD2 function leads to lack of cross-tolerance and excessive TLR responses. This study together with our previous study of NOD2 signaling 
shows that defective NOD2-induced IRF4 inhibitory function is likely to be the cause of the loss of cross-tolerance and thus the molecular basis for the role of loss of NOD2 function in CD. ${ }^{14}$

In conclusion, the present findings provide evidence that CARD15 polymorphisms function as susceptibility factors in $\mathrm{CD}$ by affecting the generation and activation of the factor, IRF4, that plays a key role in the regulation of TLR responses induced by the gut microflora. In addition, they show in studies of mice with experimental colitis that MDP administration leading to colonic IRF4 expression or intrarectal delivery of an IRF4 expression plasmid have the potential to both prevent and treat such colitis and possibly CD as well.

\section{METHODS}

Reagents. Recombinant GM-CSF and IL-4 were from Peprotech (Rocky Hill, NJ). Unless otherwise described, the doses of TLR ligands and NOD2 ligands used for stimulation were as follows: Pam3CSK4 (PAM, TLR2 ligand, $10 \mu \mathrm{g} \mathrm{ml}^{-1}$, InvivoGen, San Diego, CA), LPS (TLR4 ligand, $1 \mu \mathrm{g} \mathrm{ml}^{-1}$, Sigma, St Louis, MO), flagellin (TLR5 ligand, $1 \mu \mathrm{g} \mathrm{ml}^{-1}$, InvivoGen), CpG (TLR9 ligand, $1 \mu \mathrm{M}$, InvivoGen), and MDP (NOD2 ligand, $10 \mu \mathrm{g} \mathrm{ml}^{-1}$, InvivoGen).

Induction of colitis. TNBS colitis was induced in C57BL10 mice obtained from Japan SLC (Hamamatsu, Japan) as described previously. ${ }^{35}$ On days $-2,-1$, and 0 , mice received intraperitoneal injection of MDP $(100 \mu \mathrm{g})$ for a total of three times before intrarectal administration of $3.75 \mathrm{mg}$ of TNBS in $100 \mu \mathrm{l}$ of $45 \%$ ethanol. Mesenteric lymph node cells and colon LPMCs were isolated at the indicated time points and cultured as described previously. ${ }^{14}$ Cells were stimulated with anti-CD3 $\left(1 \mu \mathrm{g} \mathrm{ml}^{-1}\right.$, BD Pharmingen, San Jose, CA) and TLR ligands as described above. Culture supernatants were collected at 24 or $48 \mathrm{~h}$ and analyzed for cytokine production by enzyme-linked immunosorbent assay (ELISA). In some experiments, whole-cell extracts were prepared from colon tissue or LPMCs for the IP and IB analysis. In the experiments in which mice received FLAGtagged IRF4 vector ${ }^{29}$ or control vector, plasmids were encapsulated in HVJ (GenomIdea, Osaka, Japan) using protamine sulfate according to the manufacture's protocol. Then, $75 \mu \mathrm{g}$ per mouse of encapsulated plasmids were administered via the intrarectal route on days $-2,-1$, and 0 for the evaluation of colitis prevention and on day 3 for the evaluation of colitis treatment. Colon was harvested at the indicated time points. Colon tissues were stained with hematoxylin and eosin and used for the scoring of the inflammation as described previously. ${ }^{14}$ Animal use adhered to Kyoto university animal care guidelines and protocols of animal experiments were approved by the review boards of Kyoto University.

Human monocyte-derived DCs. Monocytes were isolated from the peripheral blood of healthy donors by $\mathrm{CD}_{14}{ }^{+}$microbeads (Miltenybiotec, Auburn, CA) and were cultured in 6-well plates $\left(1 \times 10^{6}\right.$ per $\left.\mathrm{ml}\right)$ in $5 \mathrm{ml}$ of complete medium (RPMI-1640 medium supplemented with $2 \mathrm{~mm}$ L-glutamine, and $10 \%$ fetal calf serum) supplemented with recombinant GM-CSF $\left(20 \mathrm{ng} \mathrm{ml}^{-1}\right)$ and recombinant IL-4 $\left(20 \mathrm{ng} \mathrm{ml}^{-1}\right)$. Ethical permission of this study was granted by the review boards of Kyoto University. After 3 days of culture, half of the medium in each well was exchanged for fresh medium. After 6 days of culture, cells $\left(1 \times 10^{6}\right.$ per ml) were incubated with MDP or medium for $24 \mathrm{~h}$ in the absence of GM-CSF and IL-4, and then washed three times and stimulated with LPS or MDP. In some experiments, cells were cultured with MG132 $\left(10 \mu \mathrm{g} \mathrm{ml}^{-1}\right.$, Enzo Life Sciences, Farmingdale, NY). In siRNA-mediated gene silencing experiments, DCs were transfected with $100 \mathrm{~nm}$ of control siRNA or IRF4 siRNA (Hs IRF4 10 FlexiTube siRNA, Qiagen, Gaithersburg, MD) by a human dendritic cell nucleofection kit (Lonza, Walkersville, MD).
Immunoprecipitation and immunoblotting. HEK293 cells (ATCC, Manassas, VA) $\left(1 \times 10^{6}\right.$ per cells) were transfected with $1 \mu \mathrm{g}$ of FLAGtagged human IRF4 vector, ${ }^{14}$ HA-tagged human MyD88, TRAF6 (InvivoGen), V5-tagged RICK, ${ }^{14}$ Myc-DDK-tagged cIAP1, MycDDK-tagged cIAP2 (Origene, Rockville, MD), HA-tagged WT Ub, K63 Ub, K48 Ub (kindly provided by Dr J. Chen), and His-tagged Ub (kindly provided by Dr H. Kondoh) by Lipofectamine 2000 (Invitrogen, Carlsbad, CA) or by Fugene 6 (Promega, Madison, WI). Mutant plasmids expressing IRF4 S447A and S448A, RICK K47A and S176A, or RICK lacking the kinase domain, intermediate domain, or CARD were created by QuickChange site-directed mutagenesis kit (Stratagene, La Jolla, CA). Whole-cell lysates were prepared 24 or $48 \mathrm{~h}$ after the transfection and were incubated with anti-FLAG conjugated beads (Sigma), anti-V5 conjugated beads (Sigma), and anti-HAconjugated beads (Sigma) overnight. In some experiments, whole-cell lysates prepared from human DCs, colonic LPMCs, and colon tissue samples were incubated with anti-K63 Ub Ab, anti-K48 Ub Ab (MBL, Nagoya, Japan) or anti-IRF4 Ab (Santa Cruz Biotechnology, Santa Cruz, CA) and protein A/G agarose (Santa Cruz Biotechnology). Anti-

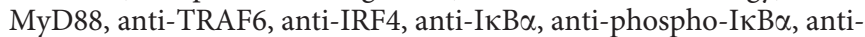
phospho-p38 anti-p38, anti-phospho-ERK, anti-ERK, and anti-His Abs were obtained from Cell Signaling Technology (CST; Danvers, MA). Anti-actin Ab (Santa Cruz), anti-RICK Ab (Cayman Chemicals, Ann Arbor, MI), anti-V5 Ab (Bethyl Laboratories, Montgomery, TX), anti-FLAG Ab (Sigma), anti-HA Ab (Sigma), and anti-Myc Ab (MBL) were used.

Immunofluorescence studies. Colon tissues were harvested and fixed in $10 \%$ formalin. Deparaffinized colon sections were then obtained and incubated with mouse anti-IRF4 Ab (Invitrogen) followed by Alexa546-conjugated anti-mouse IgG Ab (Invitrogen) by using Can Get Signal immunoreaction enhancer solution (Toyobo, Osaka, Japan) as described previously. ${ }^{43}$ For visualization of interaction between IRF4 and RICK, MyD88, and TRAF6 in colon samples, a Duolink In Situ kit was used (Olink Bioscience, Uppsala, Sweden). Goat anti-IRF4 $\mathrm{Ab}$ (Santa Cruz), rabbit anti-RICK Ab, rabbit anti-MyD88 Ab, and rabbit anti-TRAF6 Ab (Abcam, Cambridge, MA) were used to visualize target protein interactions. For visualization of interaction between IRF4 and RICK, MyD88, and TRAF6 in human DCs, cells were fixed in $4 \%$ paraformaldehyde and subjected to the protocols suggested by the Duolink In Situ kit.

ELISA. Protein concentrations of cytokines were determined by eBioscience (San Diego, CA) ELISA kits for mouse IL-12p40, mouse IL-6, and mouse tumor necrosis factor- $\alpha$. Concentration of interferon- $\gamma$ was determined by BD Bioscience (San Jose, CA) ELISA kit.

NF- $\kappa B$ activation studies. Nuclear extracts were prepared from colon tissues. Nuclear extracts were obtained with the use of a nuclear extraction kit (Active Motif, Carlsbad, CA). Binding activity of nuclear extract $(5 \mu \mathrm{g})$ to NF- $\kappa \mathrm{B}$ subunit (p50, p65, and c-Rel) was measured using a Transam kit, obtained from Active Motif, as previously described. ${ }^{43}$

Statistical analysis. Student's $t$-test was used to evaluate the significance of the differences. Statistical analysis was performed with the Prism (Graphpad software, La Jolla, CA). A value of $P<0.05$ was regarded as statistically significant.

SUPPLEMENTARY MATERIAL is linked to the online version of the paper at http://www.nature.com/mi

\section{ACKNOWLEDGMENTS}

This work was supported by Grant-in-Aid for Scientific Research (25293172, 21229009, 24229005, and 24659363) from the Japan Society for the Promotion of Science, the Japanese Society of Gastroenterology, and the Special Coordination Funds by the Ministry of Education, Culture, Sports, Science and Technology of Japan, and Astellas Pharma in Creation of Innovation Centers for Advanced Interdisciplinary Research Areas 
Program, and by the Intramural Research Program of the National Institutes of Health. We thank Ms M. Tosaka and Dr H. Ezoe for their support.

\section{DISCLOSURE}

The authors declared no conflict of interest.

c 2014 Society for Mucosal Immunology

\section{REFERENCES}

1. Strober, W. \& Watanabe, T. NOD2, an intracellular innate immune sensor involved in host defense and Crohn's disease. Mucosal Immunol. 4, 484-495 (2011).

2. Strober, W., Murray, P.J., Kitani, A. \& Watanabe, T. Signalling pathways and molecular interactions of NOD1 and NOD2. Nat. Rev. Immunol. 6, 9-20 (2006).

3. Inohara, H., Chamaillard, M., McDonald, C. \& Nunez, G. NOD-LRR proteins: role in host-microbial interactions and inflammatory disease. Annu. Rev. Biochem. 74, 355-383 (2005).

4. Abbott, D.W., Wilkins, A., Asara, J.M. \& Cantley, L.C. The Crohn's disease protein, NOD2, requires RIP2 in order to induce ubiquitinylation of a novel site on NEMO. Curr. Biol. 14, 2217-2227 (2004).

5. Abbott, D.W., Yang, Y., Hutti, J.E., Madhavarapu, S., Kelliher, M.A. \& Cantley, L.C. Coordinated regulation of Toll-like receptor and NOD2 signaling by K63-linked polyubiquitin chains. Mol. Cell. Biol. 27, 6012-6025 (2007).

6. Bertrand, M.J., Doiron, K., Labbe, K., Korneluk, R.G., Barker, P.A. \& Saleh, M. Cellular inhibitors of apoptosis CIAP1 and CIAP2 are required for innate immunity signaling by the pattern recognition receptors NOD1 and NOD2. Immunity 30, 789-801 (2009).

7. Hasegawa, M. et al. A critical role of RICK/RIP2 polyubiquitination in Nod-induced NF-kappaB activation. EMBO J. 27, 373-383 (2008).

8. Kim, Y.G., Park, J.H., Shaw, M.H., Franchi, L., Inohara, N. \& Nunez, G. The cytosolic sensors Nod1 and Nod2 are critical for bacterial recognition and host defense after exposure to Toll-like receptor ligands. Immunity $\mathbf{2 8}$, 246-257 (2008).

9. Cho, J.H. The genetics and immunopathogenesis of inflammatory bowel disease. Nat. Rev. Immunol. 8, 458-466 (2008).

10. Tada, H., Aiba, S., Shibata, K., Ohteki, T. \& Takada, H. Synergistic effect of Nod1 and Nod2 agonists with toll-like receptor agonists on human dendritic cells to generate interleukin-12 and Thelper type 1 cells. Infect. Immun. 73, 7967-7976 (2005).

11. van Heel, D.A. et al. Muramyl dipeptide and toll-like receptor sensitivity in NOD2-associated Crohn's disease. Lancet 365, 1794-1796 (2005).

12. Werts, C., Rubino, S., Ling, A., Girardin, S.E. \& Philpott, D.J. Nod-like receptors in intestinal homeostasis, inflammation, and cancer. J. Leukoc. Biol. 90, 471-482 (2011).

13. Castellaneta, A., Sumpter, T.L., Chen, L., Tokita, D. \& Thomson, A.W. NOD2 ligation subverts IFN-alpha production by liver plasmacytoid dendritic cells and inhibits their T cell allostimulatory activity via B7-H1 up-regulation. J. Immunol. 183, 6922-6932 (2009).

14. Watanabe, T. et al. Muramyl dipeptide activation of nucleotide-binding oligomerization domain 2 protects mice from experimental colitis. J. Clin. Invest. 118, 545-559 (2008).

15. Hedl, M., Li, J., Cho, J.H. \& Abraham, C. Chronic stimulation of Nod2 mediates tolerance to bacterial products. Proc. Natl. Acad. Sci. USA 104, 19440-19445 (2007).

16. Hedl, M. \& Abraham, C. Secretory mediators regulate Nod2-induced tolerance in human macrophages. Gastroenterology 140, 231-241 (2010).

17. Kullberg, BJ. et al. Crohn's disease patients homozygous for the 3020insC NOD2 mutation have a defective NOD2/TLR4 cross-tolerance to intestinal stimuli. Immunology 123, 600-605 (2008).

18. Yeretssian, G. et al. Non-apoptotic role of BID in inflammation and innate immunity. Nature 474, 96-99 (2011).

19. Meinzer, U. et al. Yersinia pseudotuberculosis effector YopJ subverts the Nod2/RICK/TAK1 pathway and activates caspase-1 to induce intestinal barrier dysfunction. Cell Host Microbe 11, 337-351 (2012).
20. Nembrini, C. et al. The kinase activity of Rip2 determines its stability and consequently Nod1 - and Nod2-mediated immune responses. J. Biol. Chem. 284, 19183-19188 (2009).

21. Dorsch, M. et al. Identification of a regulatory autophosphorylation site in the serine-threonine kinase RIP2. Cell. Signal. 18, 2223-2229 (2006).

22. Biswas, P.S. et al. Phosphorylation of IRF4 by ROCK2 regulates IL-17 and IL-21 production and the development of autoimmunity in mice. J. Clin. Invest. 120, 3280-3295 (2010).

23. Natkunam, Y., Warnke, R.A., Montgomery, K., Falini, B. \& van De Rijn, M. Analysis of MUM1/IRF4 protein expression using tissue microarrays and immunohistochemistry. Mod. Pathol. 14, 686-694 (2001).

24. Chen, Z.J. Ubiquitin signalling in the NF-kappaB pathway. Nat. Cell Biol. 7 , 758-765 (2005).

25. Tao, M., Scacheri, P.C., Marinis, J.M., Harhaj, E.W., Matesic, L.E. \& Abbott, D.W. ITCH K63-ubiquitinates the NOD2 binding protein, RIP2, to influence inflammatory signaling pathways. Curr. Biol. 19, 1255-1263 (2009).

26. Yang, Y., Yin, C., Pandey, A., Abbott, D., Sassetti, C. \& Kelliher, M.A. NOD2 pathway activation by MDP or Mycobacterium tuberculosis infection involves the stable polyubiquitination of Rip2. J. Biol. Chem. 282, 36223-36229 (2007).

27. Akira, S. \& Takeda, K. Toll-like receptor signalling. Nat. Rev. Immunol. 4, 499-511 (2004).

28. Negishi, H. et al. Negative regulation of Toll-like-receptor signaling by IRF-4. Proc. Natl. Acad. Sci. USA 102, 15989-15994 (2005).

29. Honma, K. et al. Interferon regulatory factor 4 negatively regulates the production of proinflammatory cytokines by macrophages in response to LPS. Proc. Natl. Acad. Sci. USA 102, 16001-16006 (2005).

30. Lassen, S., Lech, M., Rommele, C., Mittruecker, H.W., Mak, T.W. \& Anders, H.J. Ischemia reperfusion induces IFN regulatory factor 4 in renal dendritic cells, which suppresses postischemic inflammation and prevents acute renal failure. J. Immunol. 185, 1976-1983 (2010).

31. Xu, W.D., Pan, H.F., Ye, D.Q. \& Xu, Y. Targeting IRF4 in autoimmune diseases. Autoimmun. Rev. 11, 918-924 (2012).

32. Biswas, P.S. et al. Dual regulation of IRF4 function in Tand B cells is required for the coordination of T-B cell interactions and the prevention of autoimmunity. J. Exp. Med. 209, 581-596 (2012).

33. Mudter, J. et al. The transcription factor IFN regulatory factor-4 controls experimental colitis in mice via T cell-derived IL-6. J. Clin. Invest. 118, 2415-2426 (2008).

34. Mudter, J. et al. IRF4 regulates IL-17A promoter activity and controls RORgammat-dependent Th17 colitis in vivo. Inflamm. Bowel Dis. 17, 1343-1358 (2011).

35. Fichtner-Feigl, S., Fuss, I.J., Preiss, J.C., Strober, W. \& Kitani, A. Treatment of murine Th1 - and Th2-mediated inflammatory bowel disease with NF-kappa B decoy oligonucleotides. J. Clin. Invest. 115, 3057-3071 (2005).

36. De Vry, C.G. et al. Non-viral delivery of nuclear factor-kappaB decoy ameliorates murine inflammatory bowel disease and restores tissue homeostasis. Gut 56, 524-533 (2007).

37. Ma, A. \& Malynn, B.A. A20: linking a complex regulator of ubiquitylation to immunity and human disease. Nat. Rev. Immunol. 12, 774-785 (2012).

38. Hammer, G.E. et al. Expression of A20 by dendritic cells preserves immune homeostasis and prevents colitis and spondyloarthritis. Nat. Immunol. 12, 1184-1193 (2011).

39. Strober, W., Fuss, I. \& Mannon, P. The fundamental basis of inflammatory bowel disease. J. Clin. Invest. 117, 514-521 (2007).

40. Strober, W. \& Fuss, I.J. Proinflammatory cytokines in the pathogenesis of inflammatory bowel diseases. Gastroenterology 140, 1756-1767, e1751 (2011).

41. Wehkamp, J. et al. The Paneth cell alpha-defensin deficiency of ileal Crohn's disease is linked to Wnt/Tcf-4. J. Immunol. 179, 3109-3118 (2007).

42. Kobayashi, K.S. et al. Nod2-dependent regulation of innate and adaptive immunity in the intestinal tract. Science 307, 731-734 (2005).

43. Tsuji, Y., Watanabe, T., Kudo, M., Arai, H., Strober, W. \& Chiba, T. Sensing of commensal organisms by the intracellular sensor NOD1 mediates experimental pancreatitis. Immunity 37, 326-338 (2012). 